A Planar Comparison of Actuators for Vibration Control of Flexible Structures by

William Walker Clark

Thesis submitted to the Faculty of the Virginia Polytechnic Institute and State University in partial fulfillment of the requirements for the degree of Master of Science in

Mechanical Engineering

APPROVED:

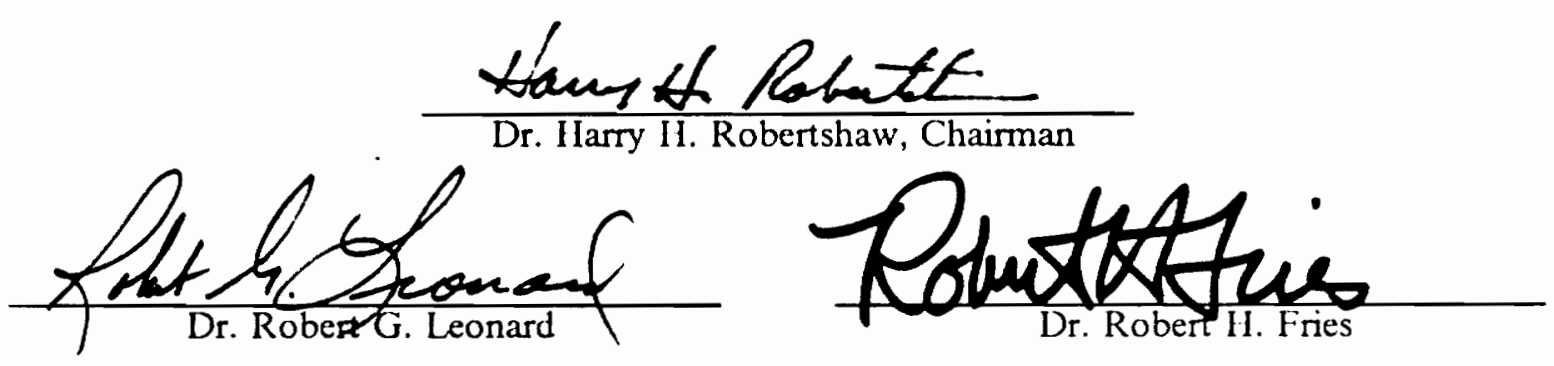

May, 1988

Blacksburg, Virginia 
20

5655

V855

1988

C.652

C. 2 


\title{
A Planar Comparison of Actuators for Vibration Control \\ of Flexible Structures
}

\author{
by \\ William Walker Clark \\ Dr. Harry H. Robertshaw, Chairman \\ Mechanical Engineering \\ (ABSTRACT)
}

Interest in large flexible space structures has grown considerably over the last decade. These distributed parameter systems exhibit vibration characteristics such as low, closely spaced natural frequencies and light damping, which, when coupled with the stringent pointing accuracy and vibration control requirements imposed on these systems, bring about interesting control problems. Addressing these problems has called for the use of active vibration control.

Up to now, two of the most popular means for active vibration control of large space structures have been proof mass and reaction wheel actuators. These actuators are inertial-type actuators in that they operate by applying forces or moments to masses whose reaction forces, imposed on the structure, act to damp the vibrations of the structure. A new class of actuators, variable geometry trusses (VGT's), has been recently introduced. These actuators are actually built into the structure, and they operate by varying their link lengths to apply forces to the structure or to change the shape of the structure itself.

This study compared the effectiveness of four actuators in controlling the planar vibrations of a cantilevered truss-beam. The actuators chosen for the study were a proof mass actuator, a reaction wheel actuator, and two VGT's, the planar truss actuator, and the planar truss proof mass actuator (a combination VGT/inertial type actuator). Numerical simulations of each beam/actuator system were performed in response to initial condition inputs. A full-state, LQR optimal feedback control law was used with each system. These simulations provided information such as time response of the closed-loop system, damping provided to the beam, and power required by each actuator. This information can be used to determine the "best" actuator for a given purpose. 
The results of these simulations show that the VGT's are preferable in terms of damping added to the beam. The proof mass actuator is more efficient as far as power required to do the control, however the efficiencies for all actuators are very similar. 


\section{Acknowledgements}

I would like to thank Dr. (Harry) Robertshaw for his encouragement and assistance throughout this work. He has provided guidance and wisdom (and quite a fews laughs) from which I have gained much and hope to gain more in the future. I would also like to thank Dr. Leonard and Dr. Fries for serving on my committee and for always being patient whenever I came knocking on their doors.

Dr. William (Bill) Patton helped a great deal with the finite element modelling in this work, and I appreciate his efforts. I would also like to acknowledge the support of Dr. Gamett Horner of NASA Langley, whose ideas were instrumental in the development of Variable Geometry Trusses as structural actuators.

There have been many people along the way who have helped me, in more ways than one, in completing this task. Thank you Brian (Dougherty), Brad (Coffey), Vince (Lovejoy), Steve (Malyevac), Brian (West), Robert (Wynn), David (Pierpont), Babak (Kimiavi), Hsiao-Feng (Kung), and, of course, the Net Apes.

I'll never be able to fully express my appreciation, but thank you Laura (Russell) for always being there. Thanks to my family, especially Mama and Daddy, for all the support and trust (and venison) you've given me over the years. And finally, I give thanks to the Good Lord above for leading me down this road and for giving me the wherewithal to see it through. 


\section{Table of Contents}

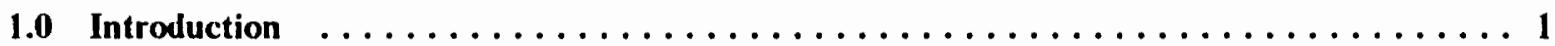

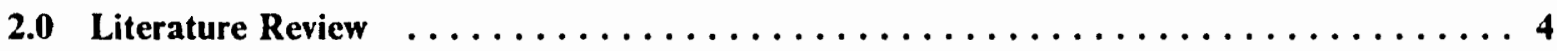

3.0 Beam Dynamic Model $\ldots \ldots \ldots \ldots \ldots \ldots \ldots \ldots \ldots \ldots \ldots \ldots \ldots$

3.1 Finite Element Model $\ldots \ldots \ldots \ldots \ldots \ldots \ldots \ldots \ldots \ldots \ldots \ldots \ldots$

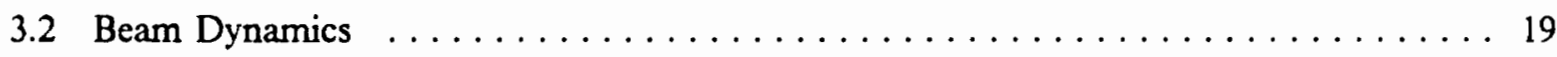

4.0 Actuator Models and System Equations $\ldots \ldots \ldots \ldots \ldots \ldots \ldots \ldots \ldots \ldots$

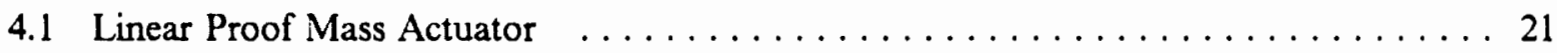

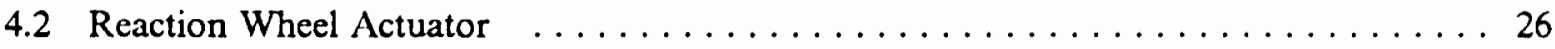

4.3 Planar Truss Actuator . . . . . . . . . . . . . . . . . . . . . . 29

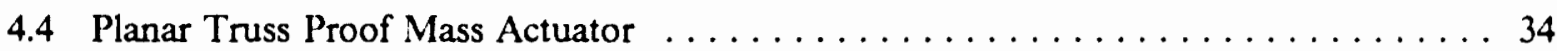

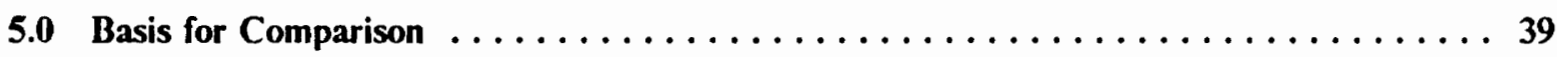

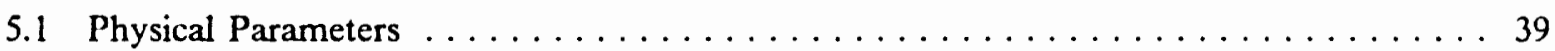

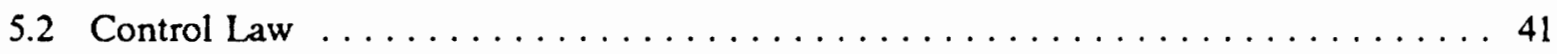

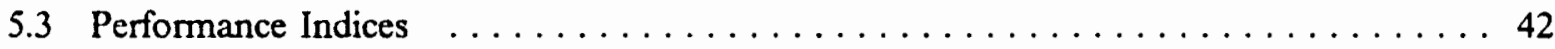

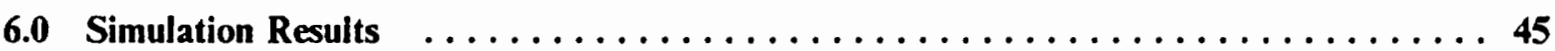

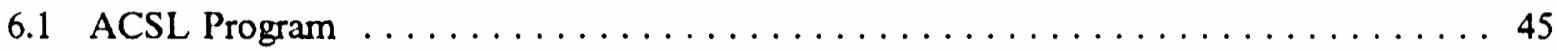

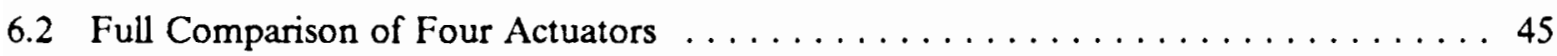

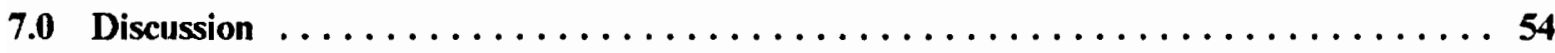


7.1 Discussion of Multiple Actuator Comparison Results $\ldots \ldots \ldots \ldots \ldots$

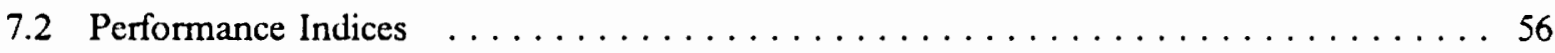

7.3 Actuator Operational Considerations $\ldots \ldots \ldots \ldots \ldots \ldots \ldots \ldots \ldots \ldots \ldots \ldots$

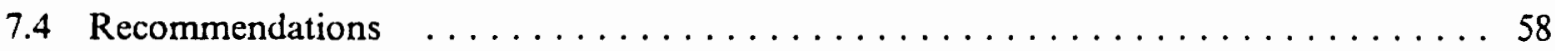

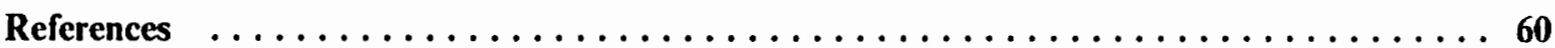

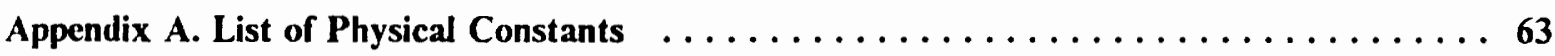

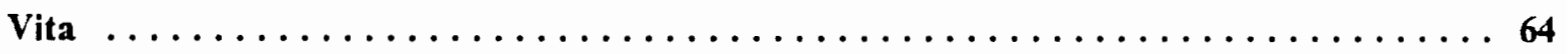




\section{List of Illustrations}

Figure 1. Illustration of $\Lambda$ ctuators Being Compared $\ldots \ldots \ldots \ldots \ldots \ldots \ldots$

Figure 2. Mode Shapes of Mini-Mast with Single Actuator Placed at Tip $\ldots \ldots \ldots \ldots$

Figure 3. Illustration of the Mini-Mast $\ldots \ldots \ldots \ldots \ldots \ldots \ldots \ldots \ldots \ldots \ldots \ldots \ldots \ldots \ldots$

Figure 4. Beam Modelled with a Single Finite Element $\ldots \ldots \ldots \ldots \ldots \ldots \ldots \ldots$

Figure 5. Beam Modelled with Two Finite Elements $\ldots \ldots \ldots \ldots \ldots \ldots \ldots \ldots \ldots$

Figure 6. Illustration of the Proof Mass Actuator $\ldots \ldots \ldots \ldots \ldots \ldots \ldots \ldots \ldots$

Figure 7 . Illustration of the Reaction Wheel Actuator $\ldots \ldots \ldots \ldots \ldots \ldots \ldots \ldots$

Figure 8 . Illustration of the Planar Truss Actuator $\ldots \ldots \ldots \ldots \ldots \ldots \ldots$

Figure 9. Illustration of the Planar Truss Proof Mass Actuator $\ldots \ldots \ldots \ldots \ldots \ldots$

Figure 10. General Shape of Beam Initial Conditions Used in Simulations . . . . . . . 46

Figure 11. Response of Mini-Mast/Proof Mass Actuator System to Initial Conditions . . . 48

Figure 12. Response of Mini-Mast/Reaction Wheel Actuator System to Initial Conditions . 49

Figure 13. Response of Mini-Mast/Planar Truss Actuator System to Initial Conditions _... 50

Figure 14. Response of Mini-Mast/Planar Truss Proof Mass Actuator System to Initial Con-

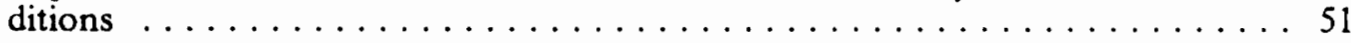

Figure 15. Motions of Planar Truss Actuator During Control $\ldots \ldots \ldots \ldots \ldots$ 


\section{List of symbols}

$\mathbf{A}$

$\mathbf{A}(t)$

$\mathbf{B}(t)$

$\mathbf{C}_{\text {octuetor }}$

$D_{i}$

E

$F(t)$

$\mathbf{F}$

$G_{p}$

$G$

I

$I_{1}$

$I_{2}$

$J$

$J_{D}$

$J_{E}$

$J_{p}$

$J_{i l}, J_{i \phi}$

$J_{\text {is }}$

$K_{B}$

$K_{r}$

$\mathbf{K}$

$\mathbf{K}_{\text {beem }}$

$\mathbf{K}_{\text {beem }}^{\prime}$
Transformation from dependent to independent coordinates

System A matrix $[$ in $\dot{\mathbf{x}}(t)=\mathbf{A}(t) \mathbf{x}(t)+\mathbf{B}(t) \mathbf{u}(t)]$

System B matrix

Actuator damping matrix

Generalized force

Beam modulus of elasticity

Motor/ballscrew applied force

Matrix of motor force coefficients

Ballscrew mechanical gain

Reaction wheel gear ratio

Mass moment of inertia of beam

Mass moment of inertia of stator

Mass moment of inertia of secondary mass

Linear Quadratic Regulator performance index

Damping performance index

Proposed Energy-LQR performance index

Actuator Power performance index

Planar truss actuator inertia constants

Ballscrew moment of inertia

Motor back emf constant

Motor torque constant

Kalman gain matrix from LQR problem

Beam stiffness matrix

Modified beam stiffness matrix (to add to planar truss actuator) 
$\mathbf{L}(x)$

$\mathscr{L}$

$M(t)$

$M_{S M}, J_{S M}$

$\mathbf{M}_{\text {actuator }}$

$\mathbf{M}_{\text {beom }}$

$\mathbf{M}_{\text {beam }}^{\prime}$

$\mathbf{M}_{\text {dep }}$

$\mathbf{M}_{\phi}, \mathbf{M}_{l}, \mathbf{M}_{p_{r}}$

$P(t)$

Pr

$\operatorname{Pr}_{T}$

$\mathbf{Q}, \mathbf{R}, \mathbf{H}$

$R_{a}$

$R_{F}$

$R_{M}$

$\mathbf{R}_{F M}$

$T_{m}$

$T(t)$

$\mathbf{T}_{1}$

$\mathbf{T}_{\phi}$

$V(t)$

$V_{m}$

$\mathbf{V}_{m}$

$X$
Interpolating polynomial

Planar truss actuator link lengths; $l_{1}, l_{2}, l_{3}$

Vector of interpolating polynomials

Lagrangian

Motor/gear applied moment

Planar truss actuator top plate mass and inertia

(Secondary mass and inertia in planar truss proof mass actuator)

Actuator mass matrix

Beam mass matrix

Modified beam mass matrix (to add to planar truss actuator)

Mass matrix in terms of dependent coordinates

Planar truss actuator mass matrices

Power consumed by the actuators

Planar truss actuator primitive coordinates; $Z_{x}, Z_{y}, \theta$

Planar truss/proof mass actuator primitive coordinates; $Z_{x}, Z_{y}, \theta, X, Y, \alpha$

Linear Quadratic Regulator penalty matrices

Motor armature resistance

Reaction force on beam from actuator

Reaction moment on beam from actuator

Vector of reaction forces on the beam from the actuators

Torque output of motor

Kinetic energy

Transformation from I to Pr coordinates

Transformation from $\varphi$ to $\operatorname{Pr}$ coordinates

Potential energy

Motor input voltage

Vector of motor input voltages

Global position coordinate ( $\hat{\mathbf{n}}_{1}$ direction) of planar truss/proof mass actuator 
Global position coordinate ( $\hat{\mathbf{n}}_{2}$ direction) of planar truss/proof mass actuator Constant of integration

Elemental stiffness matrix (for finite element model)

Finite element length

Stator mass

Secondary mass

Elemental mass as a function of axial position

Elemental mass matrix

Multiple finite element mass matrix (in terms of dependent coordinates)

Unit vector in Newtonian reference frame

Independent system coordinates

Vector of dependent coordinates for planar truss/proof mass actuator

Beam/planar truss actuator coordinates

Local elemental displacement

Vector of nodal displacements for finite element model

System state vector

Global position coordinate (rotation about $\hat{\mathbf{n}}_{3}$ ) of planar truss/proof mass actuator

Finite element nodal displacement (rotational)

Stator rotation

Secondary mass rotation

Motor shaft position

Elemental mass density

Planar truss actuator link angles; $\phi_{1}, \phi_{2}, \phi_{3}$ 


\subsection{Introduction}

Over the past decade, interest in vibration control of flexible structures has shown tremendous growth. Flexible structures can be found in many applications, but of particular interest are those to be used in space. Space structures will necessarily be made of lightweight materials so they may be launched from earth. These materials have been developed with the objective of attaining a high strength-to-weight ratio. In so doing, the materials, and the structures built from them, have little inherent structural damping. For this reason, interest has spawned in developing methods to actively control vibrations of flexible structures.

A particular area of interest is that of vibration control of iruss structures built up as long flexible beams. Many future space applications, such as th: proposed space station, incorporate such truss-beams in their main structures and appendages. There have been various methods introduced in the literature to actively damp these truss-beams. One of the primary methods being studied is the use of actuators to apply controlling forces to the beam. This paper will be concerned with four such actuators: the proof-mass actuator, the reaction wheel actuator, the planar truss actuator, and the planar truss proof-mass actuator, Fig. 1. This work describes the method and results of a study comparing the effectiveness of these actuators in controlling the planar vibrations of a particular truss-beam. The study involves simulating the response of a finite element model of a cantilevered truss-beam to initial conditions, with each of the actuators acting in turn to damp the imposed vibrations. The finite element beam model facilitates the placing of actuators at different locations on the beam, thus providing a more thorough analysis and comparison.

In this paper, a description of the beam model will be discussed, as well as descriptions of each of the actuator models. A brief discussion of the basis used for comparing the actuators will follow. The simulation results will be provided as well as a discussion of their value. 


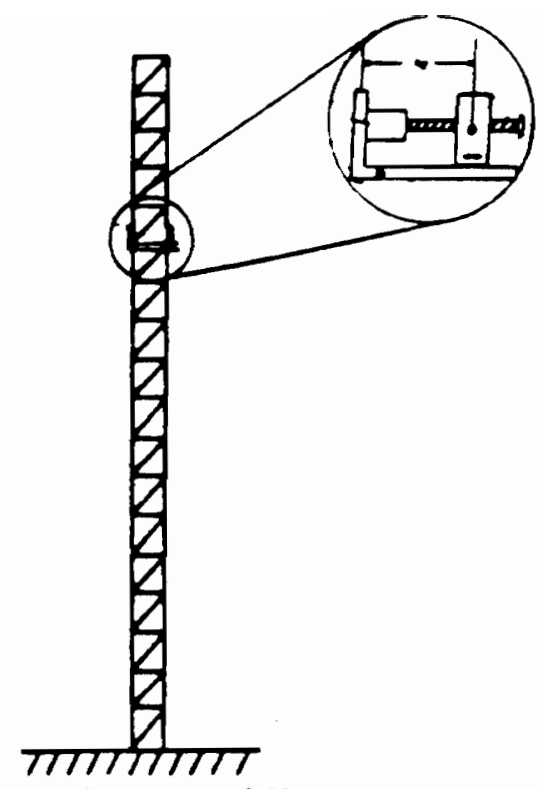

o. Linear Proof Moss

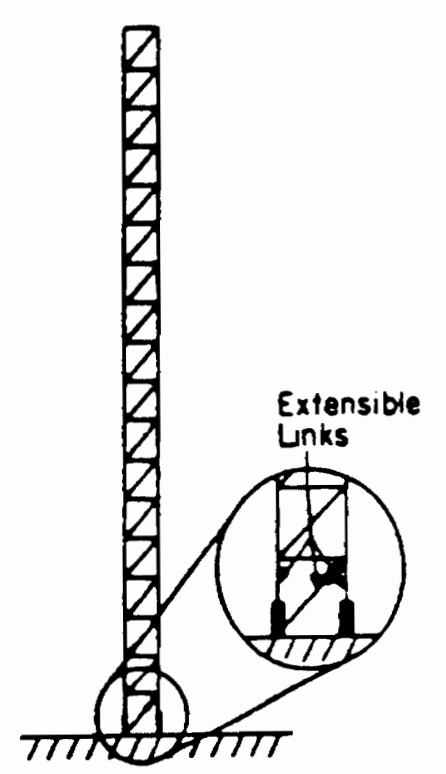

c. Plonar Truss
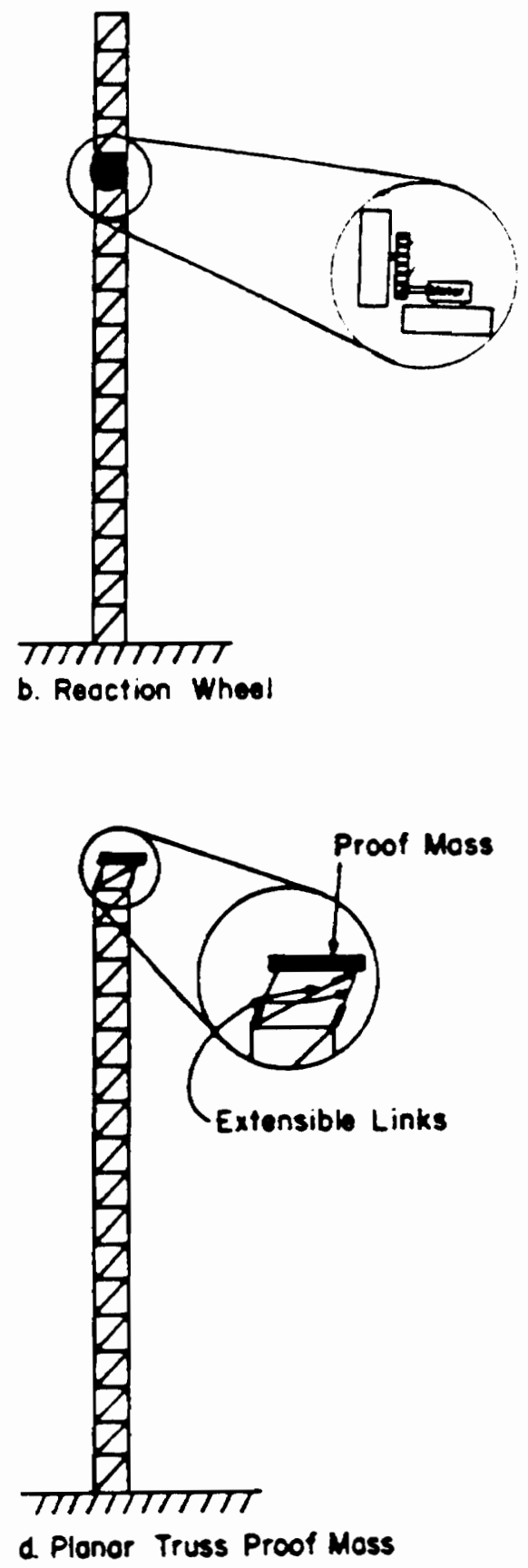

Figure 1. Illustration of Actuators Being Compared 
Choosing the "best" actuator is a difficult task because many factors are involved, but the planar comparisons contained herein will provide greater insight into choosing the "best" actuator in terms of damping capability, power requirements, and in terms of operational considerations such as weight requirements and design practicality. 


\subsection{Literature Review}

Historically, space structures have been massive, stiff, heavily damped and isolated from sources of noise and vibration. Currently the plans for space have been focused toward lighter weight and more flexible structures such as the proposed space station and Space Shuttle applications. This current thrust requires Large Space Structures (LSS) whose characteristics are very much different from space structures of the past. They are lightly damped, have lumped masses connected by long, slender members, and have low frequency modes of vibration. Not only have there been dramatic changes in structural requirements of large space structures, but increased demands on pointing precision and vibration isolation and control have brought about the need for active control of these space structures. (1)

There are several characteristics of LSS which make their study difficult. They are distributed parameter systems which are infinitely dimensional in theory, leading to high dimensional models. They have many natural frequencies which are low and many times closely spaced. Their inherent damping is very light (less than 0.5 percent). Added to the above difficulties are the stringent requirements for shape, orientation, vibration control, and pointing accuracy. (2) Based on the characteristics of LSS, there are several important areas which must be addressed in the study of vibration control of these structures.

1. An infinite dimensional system must be controlled with a finite dimensional controller.

2. The effects of unmodelled system parameters, unmodelled modes, and disturbances must be considered.

3. The effects of actuator and sensor location, number, and dynamics must be considered. (3) The first and third areas just mentioned receive major consideration in this paper because this work is concerned with different actuator configurations used in active control.

The following paragraphs review the work that has been presented in the literature concerning the development of actuators for active vibration control of flexible structures. Such a 
discussion will provide a better understanding of the purpose and results of the work described in this paper and will allow the reader to better see how this work contributes to the literature.

There are two principal ways in which to act upon a structure: by applying forces and by applying moments. This is a relatively simple concept when fixed to a large mass such as earth, but it becomes somewhat more complicated when in space because there is no longer a "ground" to react against. To accommodate this problem, the first actuators developed for vibration control of flexible structures were reaction-type (1). They produce forces and moments on the structure by using the inertial reaction forces of a small mass or rotational inertia. Perhaps the oldest and most tested of these actuators is the Control Moment Gyro or the Reaction Wheel (4). One of the first experiments in vibration control incorporating the reaction wheel actuator was performed by Lockheed (5) in which a pair of reaction wheels were placed on the end of a cantilevered beam and a proportional control law was used to add damping to the beam. This experiment was successful in adding $20 \%$ of critical damping to the beam. A slightly different configuration of the Reaction Wheel, but applying the same principle of damping, is the annular momentum control device (AMCD). In this actuator, rather than having the inertia (or reaction) wheel connected directly to the driving motor, the wheel is actually an annulus which is suspended magnetically by noncontacting magnetic stations and is driven by a noncontacting electromagnetic spin motor. The AMCD was introduced in 1975 by Anderson and Groom (6) and has since been studied for the purpose of active vibration control by others (7).

Several years after the conception of the reaction wheel actuator a new actuator was introduced, the "proof mass" actuator. This device is the translational equivalent of the reaction wheel in that it applies forces (rather than moments) to a structure using the inertial reaction forces of a small mass. Several types of proof mass actuators have been introduced. Zimmerman, Inman, and Homer (8) have developed and tested the NASA/UVA Proof Mass Actuator. Tests were conducted primarily to characterize the dynamics of the microprocessor controlled system so that those results may be included in future control work. Doanes, Waites, and Edgemon (9) have investigated the possibilities of using the linear momentum exchange device (L.MED; developed by TRW) in control of LSS. Another configuration of the proof mass actuator is the Harris COFS-I 
actuator, also referred to as a linear DC motor, or LDCM (10). Each of these actuators differs slightly in configuration. For example, some are driven with stepper motors, some use brushless dc motors, and some are driven electromagnetically, but the idea of inertia-type actuation is the same throughout.

In 1981 Mills (11) performed a theoretical study comparing the effectiveness of two inertia-type actuators, the proof mass and the reaction wheel. He modelled the actuators using dc motors to drive the inertial elements. In the study he placed the actuators at the tip of a cantilevered beam and determined each actuator's effectiveness in damping the first three modes of the beam. His results showed that for first mode the two actuators were very evenly matched, but for second and third modes, the reaction wheel actuator proved to be much superior to the proof mass actuator. The reason for the difference in control performance can be attributed in large part to actuator placement. By placing the actuators at the tip of the beam, their added mass produced a node close to that point in the beam's second and third mode shapes (Fig. 2). Placing the proof mass actuator at a node impaired its authority over second and third modes of vibration because of its translational style of actuation. The reaction wheel, however, was not affected by being placed at a node because it applies a rotational type of actuation. This paper examines the effects of placing the actuators at different locations on the beam, thus reducing the problem discussed above. With that in mind, this paper can be seen as an extension of Mills' work by studying the first two actuators in more detail as well as adding two more actuators to the comparison.

For many years reaction-type actuators such as the proof mass and reaction wheel were the only mechanical actuators being studied for LSS control. A major inadequacy of reaction-type actuators is their inability to perform pointing or slewing maneuvers. Recently, though, a class of actuators was introduced which can provide both forces and moments to the structure and accomplish slewing manuevers as well. These actuators can be classified as variable geometry trusses (VGT's) or active bay actuators. Variable geometry trusses are the newest actuators to be applied to the task of controlling vibrations of large space structures. VGT's had been studied in the past for their vibration characteristics, and for their pointing and shape control capabilities (12), but they had never been applied directly to vibration control. In 1986 Lovejoy and Robertshaw (13) showed 


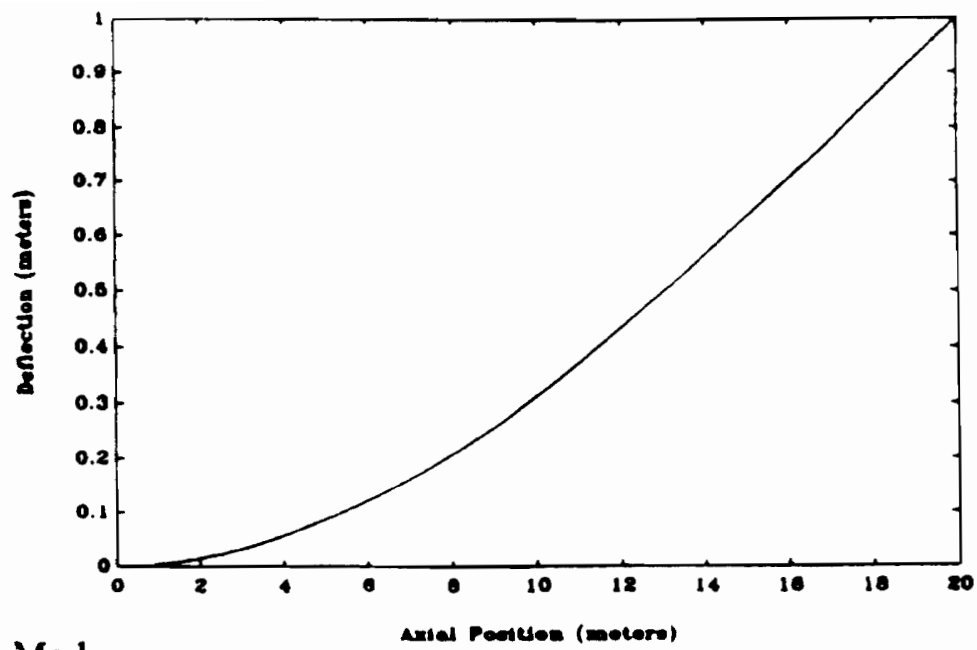

a. First Mode

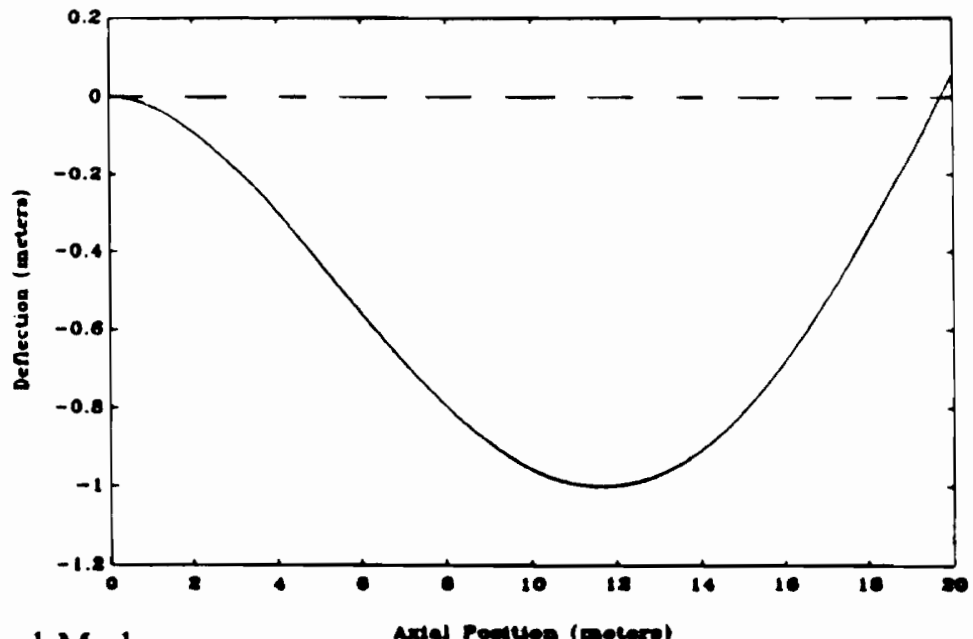

b. Second Mode

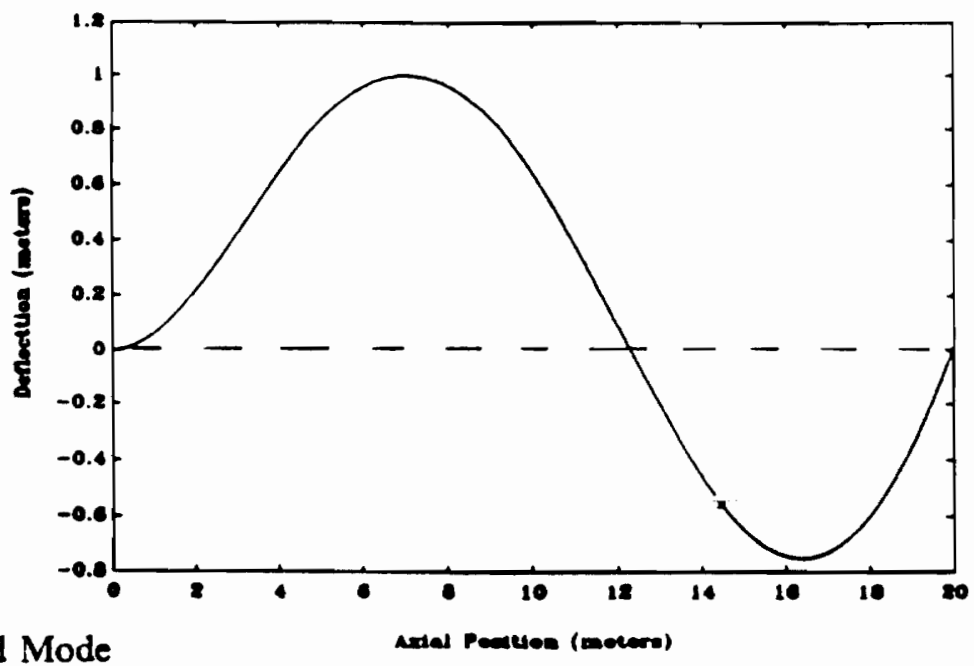

Figure 2. Mode Shapes of Mini-Mast with Single Actuator Placed at Tip 
that a planar truss actuator could be used to damp the vibrations of a beam. The truss proved to have a great deal of authority over the beam. This work has opened the possibility of threedimensional truss actuators which are built into the structure, thus eliminating the added weight of inertial elements. VGT's show more potential than any of the actuators mentioned previously, but there is still much work to be done in this area.

There are many other active control methods being studied now which are not discussed at length in this paper, but are included here to provide a complete review. One method is to use distributed piezoelectric polymers fastened to the beam which actuate the structure by applying voltages to the piezoelectric material $(14,15,16)$. This has advantages in that it is lightweight and the same material can be used for position sensing as well as actuation. The drawbacks of this method are its inability to do pointing maneuvers and the very small strains provided by the piezoelectric forces. Another relatively new method of active damping is applying thermal gradients to the structure to enhance the structures own internal damping capabilities (17). This method also has the drawback of not being able to point the structure through large motions, but it does show promise of being effective in damping vibrations. Another method of active control which has been in the literature for several years is the use of cold gas thrusters (1). The obvious disadvantage of this technology is the limited supply of gas available for actuation.

When designing a system for vibration control of large space structures, one must not be concerned only with the actuator and its dynamics, but with the control law as well. Many of the actuating methods discussed above have been tested in other experiments and simulations in the literature for the purpose of studying the growing number of control schemes. The various control laws which have been studied using the actuators described here include adaptive control (18), direct velocity feedback $(11,19)$, classical control with compensators (20), suboptimal feedback (21), and optimal control (22). A linear quadratic regulator optimal control law is used throughout this paper. 


\subsection{Beam Dynamic Model}

A major focus of this work is to simulate the planar response of a clamped-free beam to initial conditions as different actuators are applied at varying positions on the beam. The particular beam model used is a finite element model of a Bernoulli-Euler beam which has characteristics of the Mini-Mast. The Mini-Mast is a three-longeron deployable truss structure constructed by Harris Corporation for NASA Langley Research Center (Fig. 3). All members of the beam are made up of a graphite composite. Therefore the structure is lightweight and has low damping. In the model used in this work the beam is assumed to have no damping.

\subsection{Finite Element Model}

A Fortran program was written to model the linear mass and stiffness characteristics of the Mini-Mast with finite elements. The method used here is a standard finite element modelling approach and can be found in Meirovitch (23). The program breaks the beam into a variable number of finite elements. Four elements have been chosen for this study. Although the method discretizes the beam by breaking it into individual elements, each element is considered to be a continuum. Displacements at any point within the element can be described by displacements at the nodal points augmented by elemental interpolating functions. The interpolating functions chosen for this particular model are cubic splines. The equations of motion for the beam are obtained by first deriving the elemental equations of motion, then assembling those equations to arrive at the equations for the entire beam. 


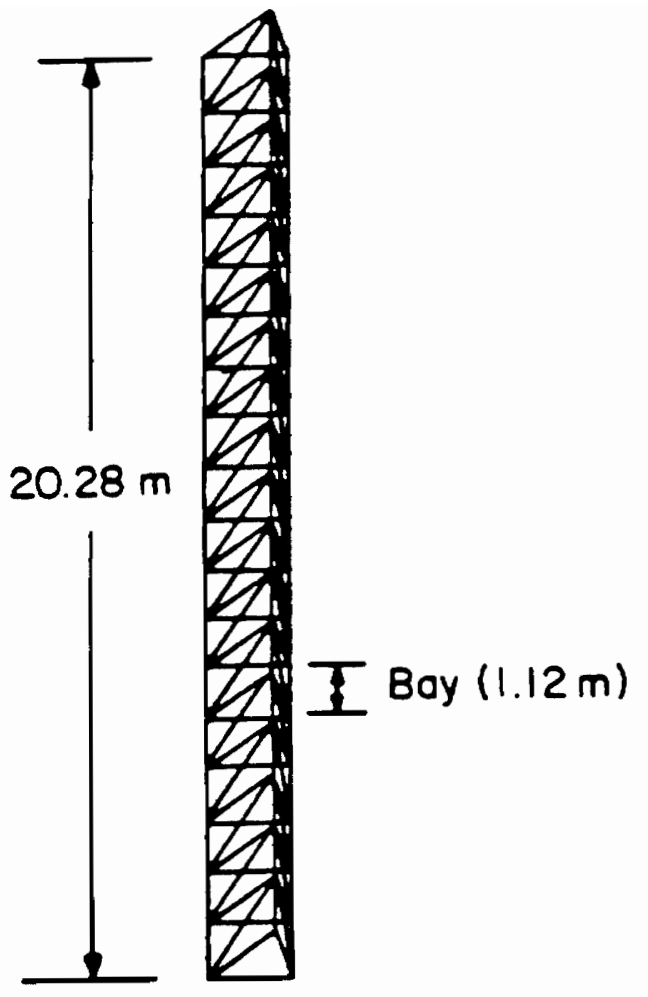

o. Side View of Mini-Mast

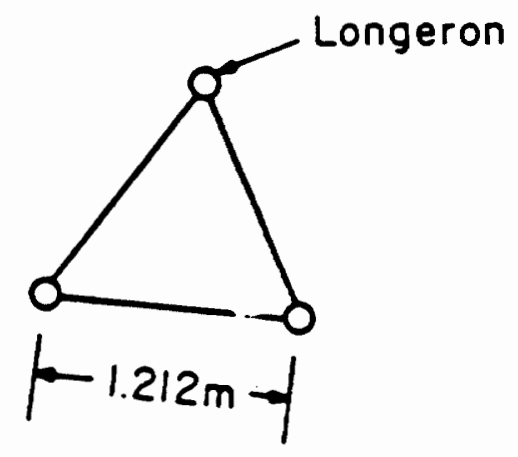

b. End View of Mini-Mast

Figure 3. Illustration of the Mini-Mast 
The elemental equations of motion are denved by a Lagrangian approach. The element's potential energy and kinetic energy are derived in terms of nodal displacements, and Lagrange's equations are applied to develop the element's equations of motion. The equations are then expressed in the form of the element's mass matrix and stiffness matrix.

Referring to Fig. 4, there are four nodal displacements for each element; $y_{1}, y_{2}, \beta_{1}$, and $\beta_{2}$. The displacement of any other point along the elcment can be described in terms of these coordinates by

$$
w(x, t)=\mathbf{L}(x)^{t} \mathbf{w}(t)
$$

where $\mathbf{L}(x)$ is the vector of interpolating polynomials and $\mathbf{w}(t)$ are the nodal displacements. Note that for an element of arbitrary length, $h$, the axial position can also be described by

$$
s=\frac{x}{h} \quad 0 \leq s \leq 1,0 \leq x \leq n
$$

The differential equation for the displacement of a Bernoulli-Luler beam can be written as

$$
E I \frac{d^{4} y(x)}{d x^{4}}=0
$$

Integrating Eq. 3.3 four times, the equation for displacement becomes

$$
y(x)=\frac{1}{6} c_{1} x^{3}+\frac{1}{2} c_{2} x^{2}+c_{3} x+c_{4}
$$

where $c_{i}^{\prime}$ s are constants of integration. The boundary conditions for this equation are

$$
\begin{gathered}
y(0)=y_{1} \\
\left.\frac{d y(x)}{d x}\right|_{x=0}=\beta_{1} \\
y(h)=\frac{1}{6} c_{1} h^{3}+\frac{1}{2} c_{2} h^{2}+c_{3} h+c_{4}=y_{2}
\end{gathered}
$$




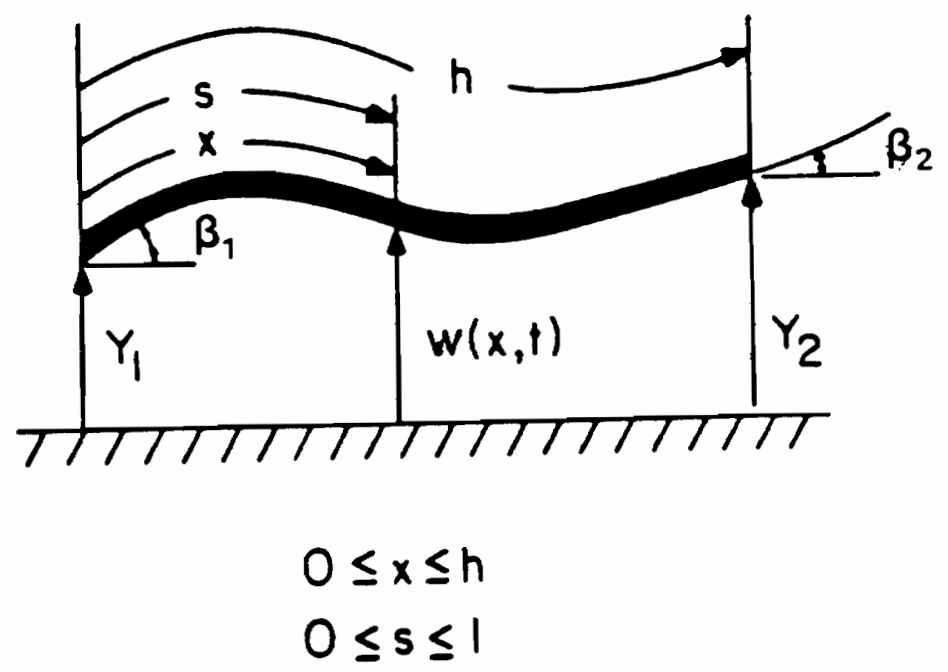

Figure 4. Beam Modelled with a Single Finite Element 


$$
\left.\frac{d y(x)}{d x}\right|_{x=h}=\frac{1}{2} c_{1} h^{2}+c_{2} h+c_{3}=\beta_{2}
$$

Applying the above boundary conditions to Eqn. 3.4 and solving, the constants of integration are found to be

$$
\begin{gathered}
c_{1}=\frac{6}{h^{3}}\left(2 y_{1}+h \beta_{1}-2 y_{2}+h \beta_{2}\right) \\
c_{2}=\frac{2}{h^{2}}\left(-3 y_{1}-2 h \beta_{1}+3 y_{2}-h \beta_{2}\right) \\
c_{3}=\beta_{1} \\
c_{4}=y_{1}
\end{gathered}
$$

Substituting into Eq. 3.4, and rearranging, the expression for bending displacement is

$$
\begin{gathered}
y(x)=\left[1-3\left(\frac{x}{h}\right)^{2}+2\left(\frac{x}{h}\right)^{3}\right] y_{1}+\left[\frac{x}{h}-2\left(\frac{x}{h}\right)^{2}+\left(\frac{x}{h}\right)^{3}\right] h \beta_{1}+ \\
{\left[3\left(\frac{x}{h}\right)^{2}-2\left(\frac{x}{h}\right)^{3}\right] y_{2}+\left[-\left(\frac{x}{h}\right)^{2}+\left(\frac{x}{h}\right)^{3}\right] h \beta_{2}}
\end{gathered}
$$

which can be written as

$$
y(x)=L_{1} y_{1}+L_{2} \beta_{1}+L_{3} y_{2}+L_{4} \beta_{2}
$$

From Eqs. 3.2 and 3.8, the interpolating polynomials are

$$
\begin{gathered}
L_{1}=2 s^{3}-3 s^{2}+1 \\
L_{2}=\left(s^{3}-2 s^{2}+s\right) h \\
L_{3}=3 s^{2}-2 s^{3} \\
L_{4}=\left(s^{3}-s^{2}\right) h
\end{gathered}
$$


The kinetic and potential energy can now be found. The kinetic energy for the beam element is defined as

$$
T(t)=\frac{1}{2} \int_{0}^{h}\left[\frac{\partial w(x, t)}{\partial t}\right]^{2} m(x) d x
$$

which, by applying Eqs. 3.1, and 3.2, can be written in the form

$$
T(t)=\frac{1}{2} h \int_{0}^{1} m(s)\left[\frac{\partial(\mathbf{L}(s) \mathbf{w}(t))}{\partial t}\right]^{2} d s
$$

or

$$
T(t)=\frac{1}{2} \dot{w}(t) t \mathbf{m} \dot{w}(t)
$$

(the $h$ appears when changing variables from $x$ to s) where

$$
\mathbf{m}=h \int_{0}^{1} m(s) \mathbf{L}(s) \mathbf{L}(s)^{t} d s
$$

Assuming the element's mass density, $\xi$, is constant along the entire length, Eq. 3.13 can be written as

$$
\mathbf{m}=\xi h \int_{0}^{1} \mathbf{L}(s) \mathbf{L}(s)^{t} d s
$$


Solving the integrals results in the mass matrix for the element

$$
\mathbf{m}=\frac{\xi h}{420}\left[\begin{array}{cccc}
156 & 22 h & 54 & -13 h \\
22 h & 4 h^{2} & 13 h & -3 h^{2} \\
54 & 13 h & 156 & -22 h \\
-13 h & -3 h^{2} & -22 h & 4 h^{2}
\end{array}\right]
$$

The potential energy for the beam element is defined as

$$
V(t)=\frac{1}{2} \int_{0}^{h} E I(x)\left[\frac{\partial^{2} w(x, t)}{\partial x^{2}}\right]^{2} d x
$$

which, by applying Eqs. 3.1 and 3.2 can be written in the form

$$
V(t)=\frac{1}{2 h^{3}} \int_{0}^{1} E I(s)\left[\frac{\partial^{2}(L(s) w(t))}{\partial s^{2}}\right]^{2} d s
$$

or

$$
V(t)=\frac{1}{2} \mathbf{w}(t)^{t} \mathbf{k} \mathbf{w}(t)
$$

(the $1 / h^{3}$ appears when changing variables from $x$ to $s$ in the integration and in the differentiation that occurs within the integrand) where

$$
\mathbf{k}=\frac{1}{h^{3}} \int_{0}^{1} E I(s) \mathbf{L}^{\prime \prime}(s) \mathbf{L}^{\prime \prime}(s)^{t} d s
$$


Assuming EI is constant along the entire length of the element, Eq. 3.18 can be written as

$$
\mathbf{k}=\frac{E I}{h^{3}} \int_{0}^{1} \mathbf{L}^{\prime \prime}(s) \mathbf{L}(s)^{\imath} d s
$$

Solving the integrals results in the stiffness matrix for the element

$$
\mathbf{k}=\frac{4 E I}{h^{3}}\left[\begin{array}{cccc}
12 & 6 h & -12 & 6 h \\
6 h & 4 h^{2} & -6 h & 2 h^{2} \\
-12 & -6 h & 12 & -6 h \\
6 h & 2 h^{2} & -6 h & 4 h^{2}
\end{array}\right]
$$

The above discussion describes the derivation of the mass and stiffness matrices for one finite element of the beam. The derivation can be extended to multiple elements by applying the same procedure for each element, then assembling all the elements to form the complete structure. The assembly process will now be described. Suppose the beam is modelled using two finite elements, Fig. 5 (four finite elements are used in the actual beam model, but for this discussion, two elements are sufficient; the assembly process remains the same for any number of elements). Each element is modelled as described above, so the result is two identical sets of mass and stiffness matrices. Figure 5 shows that in a two element model there are eight nodal coordinates used to describe the displacement of all points on the beam. Only six of these coordinates, however, are independent since the rotations and displacements of adjacent ends of the elements must be equal. A Boolean transformation can be written, then, to eliminate the redundant coordinates. Such a transformation is shown below 


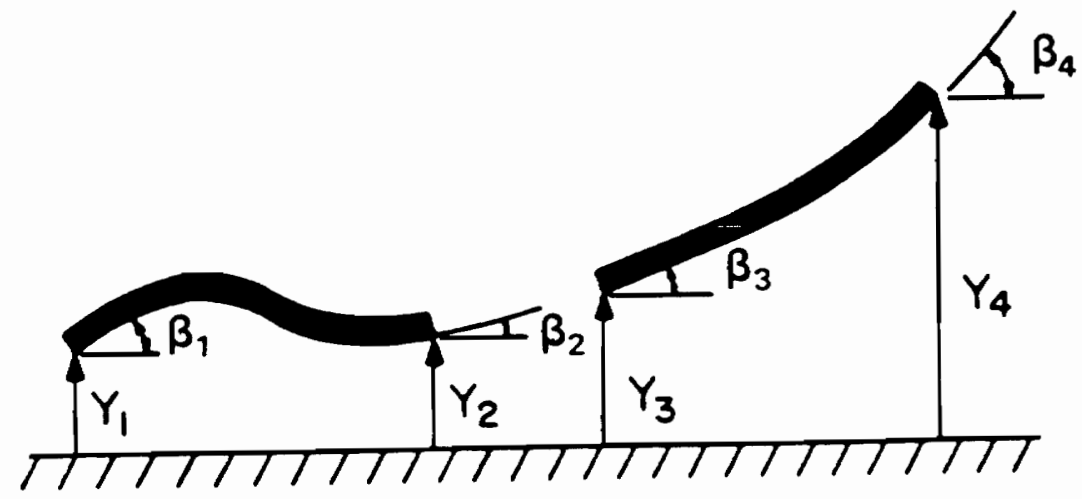

Figure 5. Beam Modelled with Two Finite Elements 


$$
\left[\begin{array}{l}
y_{1} \\
\beta_{1} \\
y_{2} \\
\beta_{2} \\
y_{3} \\
\beta_{3} \\
y_{4} \\
\beta_{4}
\end{array}\right]=\left[\begin{array}{llllll}
1 & 0 & 0 & 0 & 0 & 0 \\
0 & 1 & 0 & 0 & 0 & 0 \\
0 & 0 & 1 & 0 & 0 & 0 \\
0 & 0 & 0 & 1 & 0 & 0 \\
0 & 0 & 1 & 0 & 0 & 0 \\
0 & 0 & 0 & 1 & 0 & 0 \\
0 & 0 & 0 & 0 & 1 & 0 \\
0 & 0 & 0 & 0 & 0 & 1
\end{array}\right]\left[\begin{array}{l}
w_{1} \\
w_{2} \\
w_{3} \\
w_{4} \\
w_{5} \\
w_{6}
\end{array}\right]
$$

where $w$ are the independent nodal coordinates, $y$ are the local nodal coordinates for each element, and $\mathbf{A}$ is the Boolean transformation matrix. (The same transformation is used for velocity and acceleration.) The mass and stiffness matrices can be written in terms of the local coordinates, and then the transformation can be applied as shown below to write the kinetic and potential energies in terms of the independent nodal coordinates. (In the following discussion, the subscript, dep , means that the corresponding matrix is written in terms of dependent, coordinates.)

$$
\mathbf{m}_{\text {dep }}=\left[\begin{array}{c}
{\left[\mathbf{m}_{1}\right][0]} \\
{[0]\left[\mathbf{m}_{2}\right]}
\end{array}\right]
$$

Substituting into Eq. 3.12 gives

$$
T=\frac{1}{2} \dot{\mathbf{y}}(t)^{t} \mathbf{m}_{\text {dep }} \dot{\mathbf{y}}(t)
$$

Applying the transformation corresponding leads to

$$
T=\frac{1}{2} \dot{\mathbf{w}}(t)^{t} \mathbf{A}^{t} \mathbf{m}_{\text {dep }} \mathbf{A} \dot{\mathbf{w}}(t)
$$

which can be written as 


$$
T=\frac{1}{2} \dot{\mathbf{w}}(t)^{t} \mathbf{m} \dot{\mathbf{w}}(t)
$$

So, $\mathbf{m}$ (the mass matrix in terms of generalized coordinates) is

$$
\mathbf{m}=\mathbf{A}^{t} \mathbf{m}_{\text {dep }} \mathbf{A}
$$

Applying the same reasoning results in the generalized stiffness matrix

$$
\mathbf{k}=\mathbf{A}^{t} \mathbf{k}_{\text {dep }} \mathbf{A}
$$

The mass and stiffness matrix for the two element beam, then are written as

$$
\begin{aligned}
& \mathbf{m}_{\text {dep }}=\mathbf{A}^{t}\left[\begin{array}{c}
{\left[\mathbf{m}_{1}\right][0]} \\
{[0]\left[\mathbf{m}_{2}\right]}
\end{array}\right] \mathbf{A} \\
& \mathbf{k}_{\text {dep }}=\mathbf{A}^{t}\left[\begin{array}{c}
{\left[\mathbf{k}_{1}\right][0]} \\
{[0]\left[\mathbf{k}_{2}\right]}
\end{array}\right] \mathbf{A}
\end{aligned}
$$

where $m_{1}, m_{2}, k_{1}$ and, $k_{2}$ are the mass and stiffness matrices for the individual elements.

For a general, $n$ - element beam model there are $2 n+2$ independent coordinates needed to describe the dynamics. So the Boolean transformation matrix, A, has dimension $4 n x(2 n+2)$, and the mass and stiffness matrices for the beam are $(2 n+2) x(2 n+2)$ square, symmetric matrices.

\subsection{Beam Dynamics}

The beam model described up to this point has been free to move at both ends. In the comparison, however, the beam vibrates in clamped-free modes. This is equivalent to forcing $w_{1}$ and $w_{2}$ (the displacement and rotation of the clamped end of the beam) to be zero for all time. If 
those coordinates are always zero, then the first two rows and columns of the mass and stiffness matrix have no effect on the model and can be deleted. The order of the model has now been reduced by two degrees of freedom. So, for a four-element beam model there are ten independent nodal coordinates, two of which are always zero, leaving eight independent degrees of freedom, or a sixteenth order model for the beam.

Using $\mathbf{m}$ and $\mathbf{k}$, the Lagrangian for the beam can be written

$$
\begin{gathered}
\mathscr{L}=T-V \\
\mathscr{L}=\frac{1}{2} \dot{\mathbf{w}}(t)^{t} \mathbf{m} \dot{\mathbf{w}}(t)+\frac{1}{2} \mathbf{w}(t)^{t} \mathrm{k} \mathbf{w}(t)
\end{gathered}
$$

Lagranges equations of motion can be written as (24)

$$
\frac{d}{d t}\left[\frac{\partial \mathscr{L}}{\partial \dot{w}_{l}}\right]-\frac{\partial \mathscr{L}}{\partial w_{i}}=D_{l}
$$

where $w_{i}^{\prime}$ s are generalized coordinates and $D_{i}^{\prime} s$ are generalized forces on the beam. When the actuators are added to the beam (at the nodes), the generalized forces will be the actuator forces, and the equations of motion will become

$$
\mathbf{m} \ddot{\mathbf{w}}(t)+\mathbf{k} \mathbf{w}(t)=\mathbf{R}_{F M}
$$

where $\mathbf{R}_{F M}$ is the vector of reaction forces on the beam from the actuators. 


\subsection{Actuator Models and System Equations}

This chapter provides a derivation of the equations of motion for each of the actuators. A lumped mass approach is taken in each derivation. All actuators are assumed to have no means of storing appreciable amounts of potential energy, so potential energy is neglected in all models. Actuator parameters such as motor constants, masses of sensors, and ball screw leads are chosen so that the comparison is as fair as possible, not giving one particular actuator an unfair advantage.

The approach taken in the overall modelling is to model the actuators separately from the beam, including in each model the generalized reaction forces from the beam on the actuator or vice versa. The two systems (beam and actuator) are combined by solving for corresponding reaction forces and then setting the resulting equations equal to one another.

The following sections describe the derivations of each of the actuator equations of motion. At the end of each section the actuator model is combined with the beam model and the overall system equations of motion for that particular combination are given.

\subsection{Linear Proof Mass Actuator}

A diagram of the linear proof mass actuator is shown in Fig. 6. Mass $m_{1}$ is the fixed mass or the part of the actuator attached to the beam, and mass $m_{2}$ is the moving or "proof" mass. Throughout this thesis the proof mass will be referred to as the "secondary mass" and the fixed part of the actuator will be called the "stator". The linear proof mass actuator operates by driving the secondary mass back and forth relative to the stator, and thus imparting linear forces on the beam. 


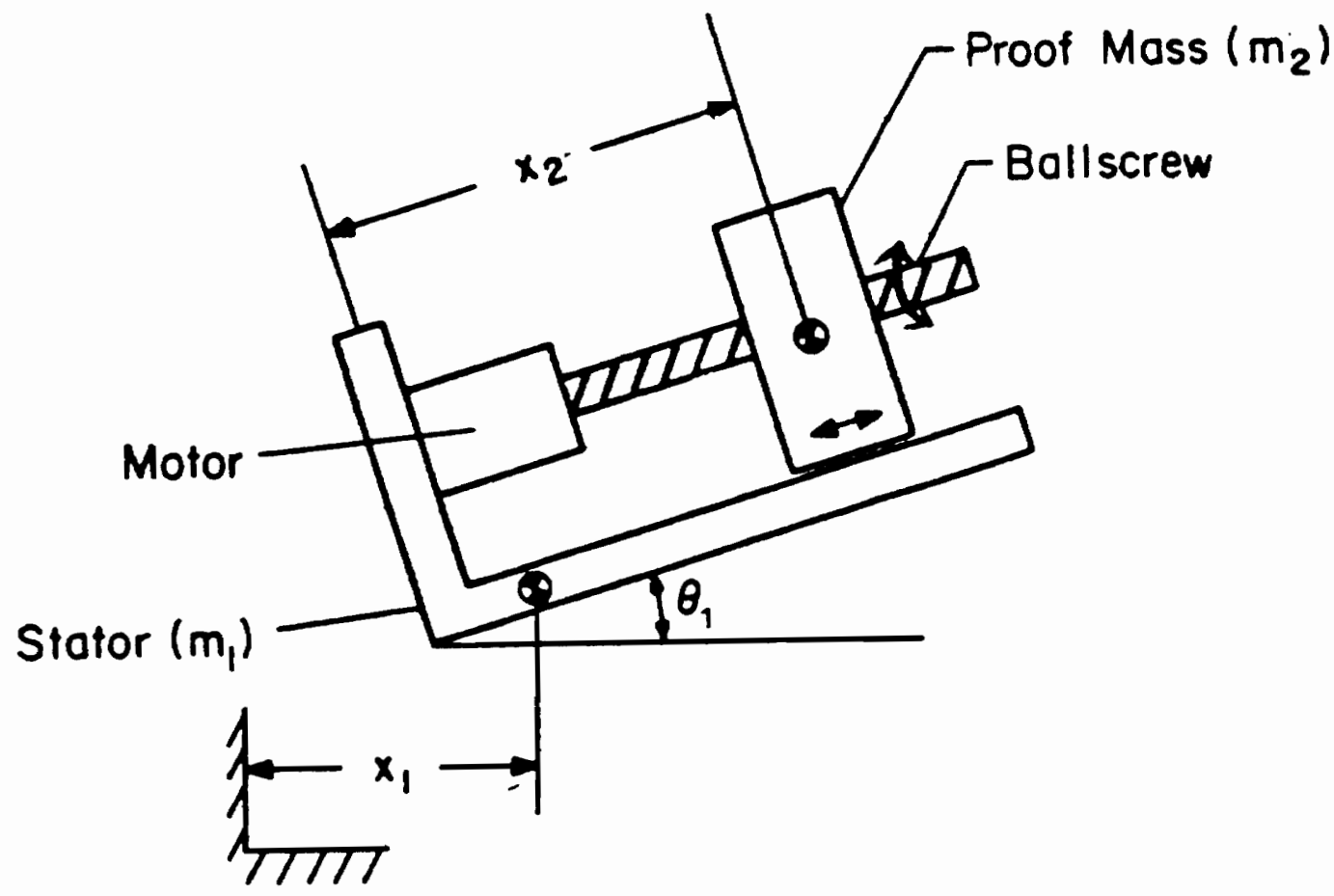

Figure 6. Illustration of the Proof Mass Actuator 
The model of the actuator chosen for the purposes of this comparison is a dc motor which drives a ball screw. The secondary mass is attached to the movable part (or nut) of the ball screw. By turning the motor, the secondary mass can be moved along the ball screw in either direction. The motor/ball screw combination applies forces on the secondary mass whose equal and opposite reactions are imparted on the beam. These forces, when applied correctly, can provide damping to the beam and thus attenuate vibrations.

It should be noted that the proof mass actuator can also operate "backwards". Inertial forces applied to the secondary mass can drive the ballscrew, and also the motor, so that the motor acts as a passive damper. When the beam moves due to its strain energy it carries the motor, ballscrew, and secondary mass with it. The inertia of the secondary mass, however, resists the motion imparted to it by the beam, so there is relative motion between the beam and the secondary mass. This relative motion can only take place if the ballscrew, and hence the motor, turn. As the motor turns, energy is transferred from the beam via the motor's back emf and is dissipated in the electrical network. Therefore, the proof mass actuator (as well as the other actuators in this comparison, since the active parts of all actuators are the same) can provide some passive damping. If the actuator design incorporated a lead screw with a high coefficient of friction instead of the ballscrew arrangement described here, or if the ballscrew gain were very low, then the beam could not drive back through the actuator, and passive damping would not be possible.

The linear proof mass actuator equations of motions will now be derived using a Lagrangian approach. The kinetic energy for the proof mass actuator, linearized to second-order terms, is, (refer to Fig. 6),

$$
T=\frac{1}{2}\left(m_{1}+m_{2}\right) \dot{x}_{1}^{2}+m_{2} \dot{x}_{1} \dot{x}_{2}+\frac{1}{2} m_{2} \dot{x}_{2}^{2}+\frac{1}{2}\left(I_{1}+I_{2}\right) \dot{\theta}_{1}^{2}+\frac{J_{l s}}{G_{p}^{2}} \dot{x}_{2}^{2}
$$

where $I_{1}$ is the mass moment of inertia of the stator, $I_{2}$ is the mass moment of inertia of the secondary mass linearized about a fixed point, $J_{l s}$ is the ballscrew mass moment of inertia, and $G_{p}$ is the ballscrew gain. The potential energy in the actuator is assumed to be negligible. From Lagranges equation, Eqs. 3.31 and 3.33, the equations of motion for the actuator become 


$$
\begin{gathered}
\left(m_{1}+m_{2}\right) \ddot{x}_{1}+m_{2} \ddot{x}_{2}=-R_{F} \\
m_{2} \ddot{x}_{1}+m_{2} \ddot{x}_{2}=F(t) \\
\left(I_{1}+I_{2}\right) \ddot{\theta}_{1}=-R_{M}
\end{gathered}
$$

where $-R_{F}$ and $-R_{M}$ are the reaction force and moment from the beam, and $F(t)$ is the generalized force from the motor/ballscrew. As mentioned earlier, the motors in all actuators are assumed to be dc armature-controlled motors which can be modelled as (neglecting the armature inductance)

$$
T_{m}=\frac{K_{T}}{R_{a}}\left(V_{m}-K_{B} \dot{\theta}_{m}\right)
$$

where $T_{m}$ is the torque output of the motor, $V_{m}$ is the input voltage, $\dot{\theta}_{m}$ is the motor shaft speed, and $K_{T}$ and $K_{B}$ are motor torque and back emf constants, respectively. If the motor is driving the secondary mass through a ballscrew, the force on the secondary mass $(F(t)$ in Eq. 4.2), considering the ballscrew gain, $G_{p}$, is

$$
F=\frac{K_{T}}{G_{p} R_{a}}\left(V_{m}-K_{B} \frac{\dot{x}_{2}}{G_{p}}\right)
$$

Substituting this force into Eq. 4.2, the equations of motion for the proof mass actuator become

$$
\begin{gathered}
\left(m_{1}+m_{2}\right) \ddot{x}_{1}+m_{2} \ddot{x}_{2}=-R_{F} \\
m_{2} \ddot{x}_{1}+m_{2} \ddot{x}_{2}+\frac{K_{T} K_{B}}{G_{p}^{2} R_{a}} \dot{x}_{2}=\frac{K_{T} V_{m}}{G_{p} R_{a}} \\
\left(I_{1}+I_{2}\right) \ddot{\theta}_{1}=-R_{M}
\end{gathered}
$$

Now the linear proof mass actuator and the beam will be combined to form a complete system. In this derivation, the actuator will be added at the tip of the beam, which corresponds to 
the last node in the finite element model. In Eq. 4.5, $x_{1}$ is the generalized coordinate for the actuator stator motion. This is also the coordinate describing the linear motion of the node on the beam at which the actuator is attached. With this redundancy of coordinates in mind, the mass and damping matrices for the actuator can be augmented with zeros to take into account the other beam coordinates in the final equation. The mass and damping matrices for the actuator become

$$
\begin{aligned}
& M_{\text {actuator }}=\left[\begin{array}{ccccc}
0 & \ldots & 0 & 0 & 0 \\
\cdot & & \cdot & \cdot & \cdot \\
\cdot & & \cdot & \cdot & \cdot \\
\cdot & & \cdot & \cdot & \cdot \\
0 & \ldots & m_{1}+m_{2} & 0 & m_{2} \\
0 & \ldots & 0 & I_{1}+I_{2} & 0 \\
0 & \ldots & m_{2} & 0 & m_{2}
\end{array}\right] \\
& C_{\text {acruator }}=\left[\begin{array}{ccccc}
0 & \ldots & 0 & 0 & 0 \\
\cdot & & \cdot & \cdot & \cdot \\
\cdot & & \cdot & \cdot & \cdot \\
\cdot & & \cdot & \cdot & \cdot \\
0 & \ldots & 0 & 0 & 0 \\
0 & \ldots & 0 & 0 & 0 \\
0 & \ldots & 0 & 0 & \frac{K_{T} K_{B}}{G_{p}^{2} R_{a}}
\end{array}\right]
\end{aligned}
$$

where both matrices are $9 \times 9$. Note that moving the actuator to another node on the beam only requires moving the terms $m_{1}+m_{2}, l_{1}+I_{2}$, and the two off-diagonal $m_{2}$ terms to the proper node locations in the mass matrix. Adding an actuator to the system can be done by simply adding the necessary mass and rotational inertia terms to the mass matrix, and adding another damping term to the damping matrix. Note that adding an actuator adds one independent coordinate to the system. 
Solving for the reaction forces and setting the beam and actuator equations equal to one another, the overall system equations become

$$
\left(\mathbf{M}_{\text {beam }}+\mathbf{M}_{\text {actuator }}\right) \ddot{\mathbf{q}}+\mathbf{C}_{\text {acruator }} \dot{\mathbf{q}}+\mathbf{K}_{\text {beam }} \mathbf{q}=\mathbf{F V}_{m}
$$

where $q$ consists of the beam nodal displacements and rotations and the proof mass displacement, $\mathbf{F}$ is the matrix of motor force coefficients, and $\mathbf{V}_{m}$ is the vector of motor input voltages (scalar for one actuator).

\subsection{Reaction Wheel Actuator}

A diagram of the reaction wheel actuator is shown in Fig. 7. Inertia $I_{1}\left(m_{1}\right)$ is the stator and inertia $I_{2}\left(m_{2}\right)$ is the moving part or reaction wheel. The reaction wheel actuator operates by spinning the reaction wheel about an axis which is fixed on the beam, thus imparting moments on the beam. Once again, the model of the reaction wheel actuator incorporates a dc motor which drives the reaction wheel through a gear. The gear is included to provide the reaction wheel actuator with the same mechanical advantage that the linear proof mass actuator attains from the ballscrew gain. By driving the motor, the reaction wheel is accelerated through some angle $\theta_{2}$. Thus the motor/gear combination applies moments to the reaction wheel whose equal and opposite reaction moments are imparted on the beam. These moments work to attenuate vibrations of the beam.

At this time an appropriate model for the reaction wheel actuator will be presented. The second-order linearization of kinetic energy for the reaction wheel is

$$
T=\frac{1}{2} \dot{\theta}_{1}^{2} I_{1}+\frac{1}{2}\left(\dot{\theta}_{1}+\dot{\theta}_{2}\right)^{2} I_{2}+\frac{1}{2}\left(m_{1}+m_{2}\right) \dot{x}_{1}^{2}
$$

The potential energy is again assumed to be negligible. Applying Eqs. 3.31 and 3.33, the equations of motion for the actuator become 


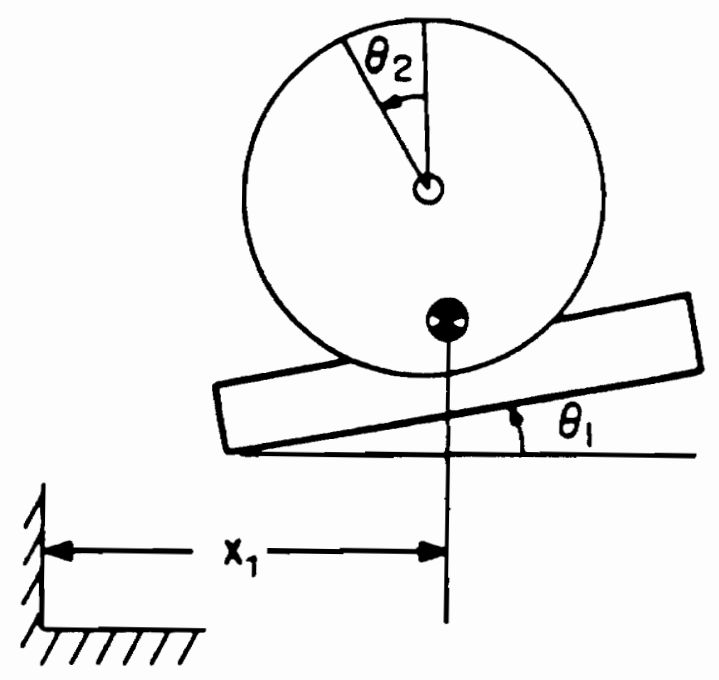

a. End View of Reaction Wheel Actuctor

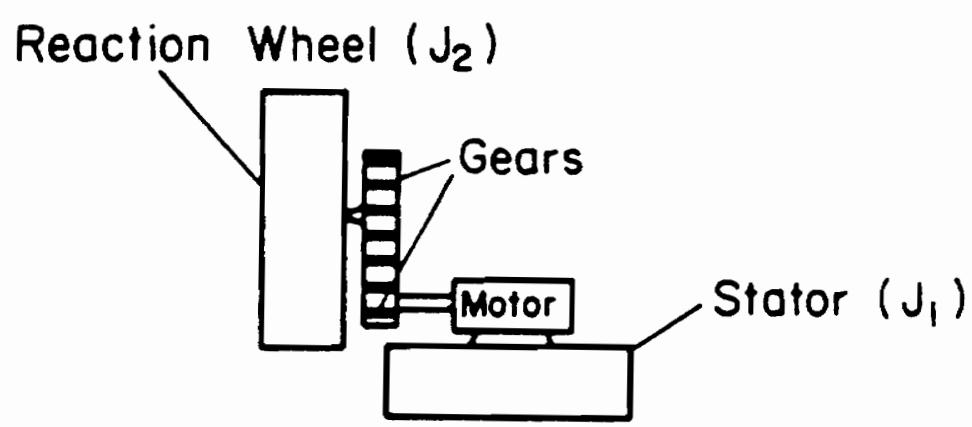

b. Side View of Reaction Wheel Actuator

Figure 7. Illustration of the Reaction Wheel Actuator 


$$
\begin{gathered}
\ddot{\theta}_{1}\left(I_{1}+I_{2}\right)+\ddot{\theta}_{2} I_{2}=-R_{M} \\
\ddot{\theta}_{2} I_{2}+\ddot{\theta}_{1} I_{2}=M(t) \\
\left(m_{1}+m_{2}\right) \ddot{x}_{1}=-R_{F}
\end{gathered}
$$

where $-R_{M}$ and $-R_{F}$ are the reaction moment and force from the beam, and $M(t)$ is the generalized moment from the motor/gear. Using the same model for the motor as with the linear proof mass actuator (Eq. 4.3), and substituting for $M(t)$, the equations of motion for the reaction wheel actuator become

$$
\begin{gathered}
\ddot{\theta}_{1}\left(I_{1}+I_{2}\right)+\ddot{\theta}_{2} I_{2}=-R_{M} \\
\left(\ddot{\theta}_{2}+\ddot{\theta}_{i}\right) I_{2}+\frac{K_{T} K_{B}}{G_{r}^{2} R_{a}} \dot{\theta}_{2}=\frac{K_{T}}{G_{r} R_{a}} V_{m} \\
\left(m_{1}+m_{2}\right) \ddot{x}_{1}=-R_{F}
\end{gathered}
$$

where $G_{r}$ is the gear ratio found in the reaction wheel actuator.

Now the reaction wheel actuator and the beam will be combined to form a complete system. As with the linear proof mass actuator, $\theta_{1}$ in Eq. 4.10 is the generalized coordinate for the motion of the actuator stator and also the coordinate describing the rotary motion of the node on the beam where the actuator is attached. Realizing that the redundant coordinate in this case is a rotational and not a displacement coordinate, the mass and damping matrices for the reaction wheel actuator have the same form as those for the linear proof mass actuator. The augmented mass and damping matrices for the reaction wheel actuator become 


$$
\begin{aligned}
& M_{\text {acruator }}=\left[\begin{array}{ccccc}
0 & \ldots & 0 & 0 & 0 \\
\cdot & & \cdot & \cdot & \cdot \\
\cdot & & \cdot & \cdot & \cdot \\
. & & . & \cdot & \cdot \\
0 & \ldots & 0 & m_{1}+m_{2} & 0 \\
0 & \ldots & I_{1}+I_{2} & 0 & I_{2} \\
0 & \ldots & I_{2} & 0 & I_{2}
\end{array}\right] \\
& C_{\text {actuator }}=\left[\begin{array}{ccccc}
0 & \ldots & 0 & 0 & 0 \\
\cdot & & \cdot & \cdot & \cdot \\
\cdot & & \cdot & \cdot & \cdot \\
\cdot & & \cdot & \cdot & \cdot \\
0 & \ldots & 0 & 0 & 0 \\
0 & \ldots & 0 & 0 & 0 \\
0 & \ldots & 0 & 0 & \frac{K_{T} K_{B}}{G_{r}^{2} R_{a}}
\end{array}\right]
\end{aligned}
$$

where, again, both matrices are $9 \times 9$. Note that adding an actuator to this system follows the same logic as that presented for the proof mass actuator, and once again, adding an actuator adds one degree of freedom to the system.

Solving for reaction moments and setting the beam and actuator equations equal to one another, the overall system equations become the same form as Eq. 4.7.

\subsection{Planar Truss Actuator}

The planar truss actuator is shown in Fig. 8. This actuator, made up of three extensible links, is located between the clamped end of the beam and ground in this study. The extensible 


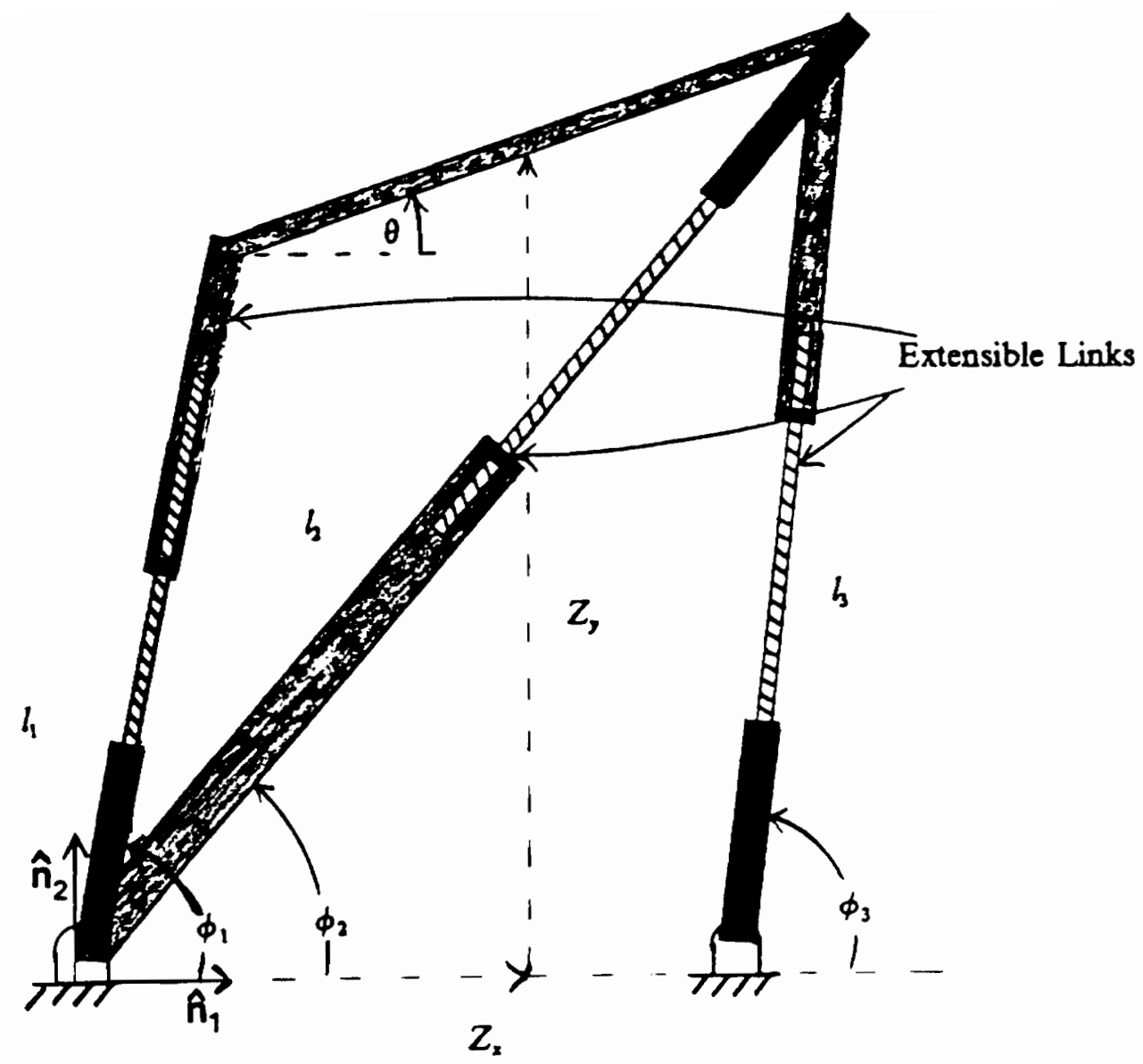

Figure 8. Illustration of the Planar Truss Actuator 
links are eac's made up of motor/ballscrew combinations much like the linear proof mass actuator. The fixed ends of the ballscrews are attached to ground, and the ballscrew nuts are attached to the beam. As the motors turn the ballscrews, the beam is moved back and forth in the direction of the nut motion. As can be seen from the extensible link arrangement in Fig. 8, the planar truss actuator has control over three degrees of freedom of the base of the beam, $Z_{x}, Z_{y}$, and $\theta$. By controlling the motors correctly, the reaction forces, $R_{x}, R_{y}$, and $M_{\theta}$, imparted on the base of the beam work to attenuate the beam vibrations.

The derivation of the actuator equations of motion will follow the same method as used by Lovejoy [18]. The actuator is separated into individual links, the energies are written for each, and then summed. The second-order linearization of kinetic energy for links 1,2, and 3 is

$$
T_{l}=\frac{1}{2}\left(J_{l \phi}+m_{i}\left(l_{l}-r_{l}\right)^{2}\right) \dot{\phi}_{l}^{2}+\frac{1}{2} J_{l l} i_{i}^{2} \quad i=1,2,3
$$

The energy for link 4 , the top plate of the actuator where the beam is attached, is

$$
T_{4}=\frac{1}{2} M_{S M}\left(\dot{Z}_{x}^{2}+\dot{Z}_{y}^{2}\right)+\frac{1}{2} J_{S M} \dot{\theta}^{2}
$$

Potential energy is neglected in this model because the actuator is assumed to have no means of storing appreciable potential energy. Note that three dependent coordinate systems have been used in deriving the kinetic energy, $\operatorname{Pr},\left(\left[Z_{x} Z_{y} \theta\right]^{t}\right), I,\left(\left[l_{1} l_{2} l_{3}\right]^{t}\right)$, and $\varphi,\left(\left[\phi_{1} \phi_{2} \phi_{3}\right]^{t}\right)$. The kinetic energy can be expressed in terms of these three systems as

$$
T=\dot{\varphi}^{t} \mathbf{M}_{\phi} \dot{\varphi}+\dot{i}^{t} \mathbf{M}_{\dot{\boldsymbol{j}}}+\dot{\mathbf{P r}}^{t} \mathbf{M}_{P_{r}} \dot{\mathbf{P r}}
$$

Coordinate transformations must be introduced to express the energy in one system basis. The "primitive" system, $\operatorname{Pr}$, has been chosen as the basis for convenience. Lovejoy derived these coordinate transformations and they are presented here as:

$$
I=T_{l} \operatorname{Pr}
$$


where

$$
\begin{gathered}
\mathrm{T}_{l}= \\
{\left[\begin{array}{ccc}
\frac{Z_{x}-d \cos \theta}{l_{1}} & \frac{Z_{y}-d \sin \theta}{l_{1}} & \frac{Z_{x} d \sin \theta-Z_{y} d \cos \theta}{l_{1}} \\
\frac{Z_{x}+d \cos \theta}{l_{2}} & \frac{Z_{y}+d \sin \theta}{l_{2}} & \frac{Z_{y} d \cos \theta-Z_{x} d \sin \theta}{l_{2}} \\
\frac{Z_{x}+d \cos \theta-2 d}{l_{3}} & \frac{Z_{y}+d \sin \theta}{l_{3}} & \frac{Z_{y} d \cos \theta-Z_{x} d \sin n+2 d^{2} \sin \theta}{l_{3}}
\end{array}\right]}
\end{gathered}
$$

and

$$
\varphi=\mathrm{T}_{\phi} \operatorname{Pr}
$$

where

$$
\begin{gathered}
\mathbf{T}_{\phi}= \\
{\left[\begin{array}{ccc}
\frac{-\left(Z_{y}-d \sin \theta\right)}{l_{1}^{2}} & \frac{-\left(Z_{x}-d \cos \theta\right)}{l_{1}^{2}} & \frac{-\left(Z_{x}-d \cos \theta\right) d \cos \theta-\left(Z_{y}-d \sin \theta\right) d \sin \theta}{l_{1}^{2}} \\
\frac{-\left(Z_{y}+d \sin \theta\right)}{l_{2}^{2}} & \frac{-\left(Z_{x}+d \cos \theta\right)}{l_{2}^{2}} & \frac{\left(Z_{y}+d \sin \theta\right) d \sin \theta+\left(Z_{x}+d \cos \theta\right) d \cos \theta}{l_{2}^{2}} \\
\frac{-\left(Z_{y}+d \sin \theta\right)}{l_{3}^{2}} & \frac{-\left(Z_{x}+d \cos \theta-2 d\right)}{l_{3}^{2}} & \frac{\left(Z_{y}+d \sin \theta\right) d \sin \theta+\left(Z_{x}+d \cos \theta-2 d\right) d \cos \theta}{l_{3}^{2}}
\end{array}\right]}
\end{gathered}
$$

These transformations are exact for velocities as well, so it is true to say

$$
\begin{gathered}
\dot{i}=\mathrm{T}_{l} \dot{\mathrm{Pr}} \\
\dot{\varphi}=\mathrm{T}_{\phi} \dot{\mathrm{Pr}}
\end{gathered}
$$


For this work it is also assumed that

$$
\begin{gathered}
\ddot{\mathrm{j}}=\mathbf{T}_{l} \ddot{\mathbf{P r}} \\
\ddot{\varphi}=\mathrm{T}_{\phi} \ddot{\mathrm{Pr}}
\end{gathered}
$$

Using the above transformations, the kinetic energy can be written as

$$
T=\dot{\mathbf{P r}^{t}} \mathbf{M}_{\text {acruator }} \dot{\mathbf{P r}}
$$

where

$$
\mathbf{M}_{\text {acruator }}=\left(\mathbf{T}_{\phi}^{t} \mathbf{M}_{\phi} \mathbf{T}_{\phi}+\mathbf{T}_{l}^{t} \mathbf{M}_{l} \mathbf{T}_{l}+\mathbf{M}_{\mathrm{Pr}}\right)
$$

Using the above expressions for kinetic energy and applying Eqs. 3.31 and 3.33, Lagrange's equations, the planar truss actuator equations of motion become

$$
2 \mathbf{M}_{\text {actuator }} \ddot{\mathbf{P r}}=-\mathbf{R}_{F M}+\mathrm{I}_{l} \mathbf{F V} \mathbf{V}_{m}
$$

where $-\mathbf{R}_{F M}$ is the vector of reaction forces and moments from the beam, and $\mathbf{T}_{i} \mathbf{F V} \mathbf{m}_{m}$ is the vector of generalized forces from the motor/ballscrews. Applying the model for the motors developed earlier, the equations of motion for the planar truss actuator become

$$
2 \mathbf{M}_{b} \ddot{\mathbf{P r}}+\frac{K_{T} K_{b}}{G_{p}^{2} R_{a}} \mathbf{T}_{l}^{t} \mathbf{T}_{l} \dot{\mathbf{P r}}=-\mathbf{R}_{F M}+\frac{K_{T}}{G_{p} R_{a}} \mathbf{T}_{l}^{t} \mathbf{V}_{m}
$$

At this time, the beam and actuator will be combined to form a complete system. Before doing so, it is important to note that in this case, the base of the beam undergoes motion through three degrees of freedom, whereas in the previous case, the base of the beam was constrained to remain motionless. So, before combining the two models, three coordinates must be added to the existing beam model. These coordinates are the same as the primitive coordinates of the actuator model. The beam no longer vibrates in clamped-free modes because the base is free to move 
through the coordinates $Z_{x}$, and $\theta$, and the entire beam can undergo rigid body motion through the coordinate $Z_{y}$. Taking into account this difference, the beam equations of motion can be derived to be

$$
\mathbf{M}_{\text {beam }}^{\prime} \ddot{q}^{\prime}+\mathbf{K}_{\text {beam }}^{\prime} \mathbf{q}^{\prime}=\mathbf{R}_{F M}
$$

where the primes distinguish this beam model from the one used in the previous two sections. The vector of coordinates for this model is

$$
\mathbf{q}^{\prime}=\left\{\begin{array}{llllllll}
Z_{x} & Z_{y} & \theta & y_{1} & \theta_{1} & \ldots & y_{4} & \theta_{4}
\end{array}\right\}^{t}
$$

where $y_{i}$ and $\theta_{i}(i=1,2,3,4)$ are the local nodal displacements and rotations, respectively.

The beam and actuator models can be combined to form the total system equations of motion by first augmenting the beam equations by adding zeros to account for the other coordinates, solving both sets of equations for the reaction forces, and setting them equal to one another. The overall system equations of motion are

$$
\left(\mathbf{M}_{\text {beam }}^{\prime}+\mathbf{M}_{\text {actuator }}\right) \ddot{\mathbf{q}}^{\prime}+\mathbf{C}_{\text {actuator }} \dot{\mathbf{q}}^{\prime}+\mathbf{K}_{\text {beam }} \mathbf{q}^{\prime}=F V_{m}
$$

\subsection{Planar Truss Proof Mass Actuator}

A diagram of the planar truss proof mass actuator is shown in Fig. 9. This actuator is configured the same as the planar truss actuator, it is made up of three extensible links consisting of motor/ballscrew combinations, but it operates somewhat differently. In this case the actuator is placed at the tip of the beam and operates as an inertia-type actuator. It is not fixed to ground as the planar truss actuator is. When the motors turn the ballscrews, the secondary mass is accelerated through three coordinates, $Z_{x}, Z_{y}$, and $\theta$, by forces $R_{x}, R_{y}$, and $M_{\theta}$, whose equal and opposite 


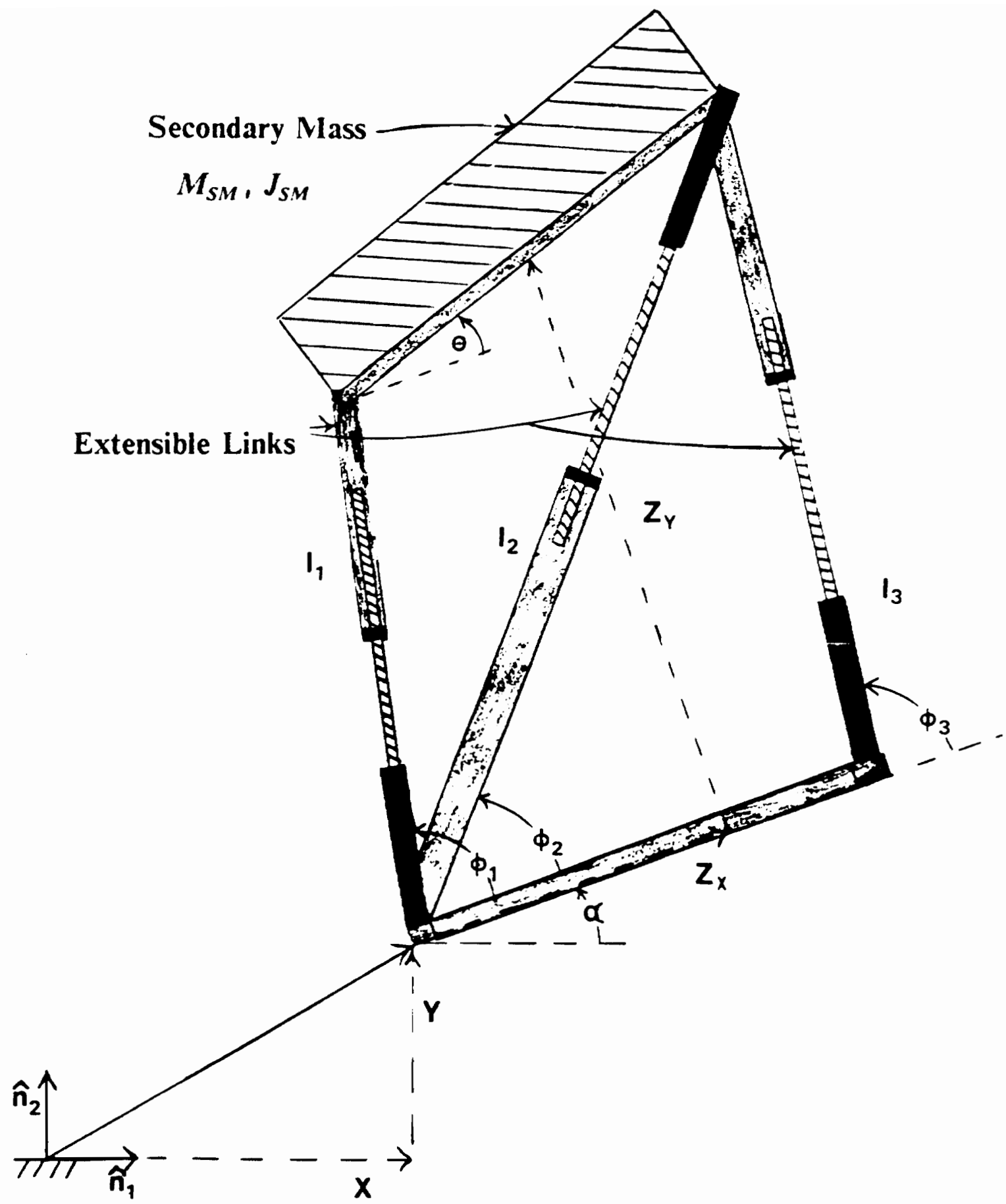

Figure 9. Illustration of the Planar Truss Proof Mass Actuator 
reactions are applied to the beam. These reaction forces, when applied correctly, work to attenuate vibrations in the beam.

The derivation of the equations of motion of the planar truss proof mass actuator will follow the same method as in the previous section, with the added complication that the actuator is no longer fixed to ground. The base of the actuator can move through some displacements $\mathrm{x}$, $y$, and $\alpha$, the motions of the tip of the beam. Since motion in the y-direction is negligible and has been neglected in the finite element model, it will be neglected in this model as well. Considering these global motions superimposed on the local actuator motions discussed in the last section the actuator equations of motion will now be derived.

The bi-linearized kinetic energy for the planar truss proof mass actuator can be written as,

$$
T=\dot{\mathbf{q}}_{T}(t)^{t} \mathbf{M}_{\text {dep }} \dot{\mathbf{q}}_{T}(l)
$$

where

$$
\mathbf{q}_{T}=\left\{\begin{array}{llllllllllllll}
x & \alpha & \phi_{1} & \phi_{2} & \phi_{2} & l_{1} & l_{2} & l_{3} & Z_{x} & Z_{y} & 0
\end{array}\right\}^{l}
$$

and $\mathbf{M}_{\text {dep }}$ is the mass matrix for the actuator written in terms of dependent coordinates. Note that the vector of coordinates, $q_{T}$, has increased by two elements ( $x$ and $\alpha$; the rigid body motions of the actuator) over that used in the model of the planar truss actuator. Once again, potential energy is neglected in the actuator.

Before applying Lagrange's equations, the kinetic energy must be written in terms of independent coordinates. The above set of equations describes the actuator with 11 coordinates where only 5 are independent. The coordinate transformation matrices, $T_{l}$ and $T_{\phi}$, introduced in the previous section will be used to write the equations in terms of 5 "primitive" coordinates. The following transformation from dependent to primitive coordinates will be used:

$$
\mathbf{q}_{T}=\mathbf{A} \operatorname{Pr}_{T}
$$




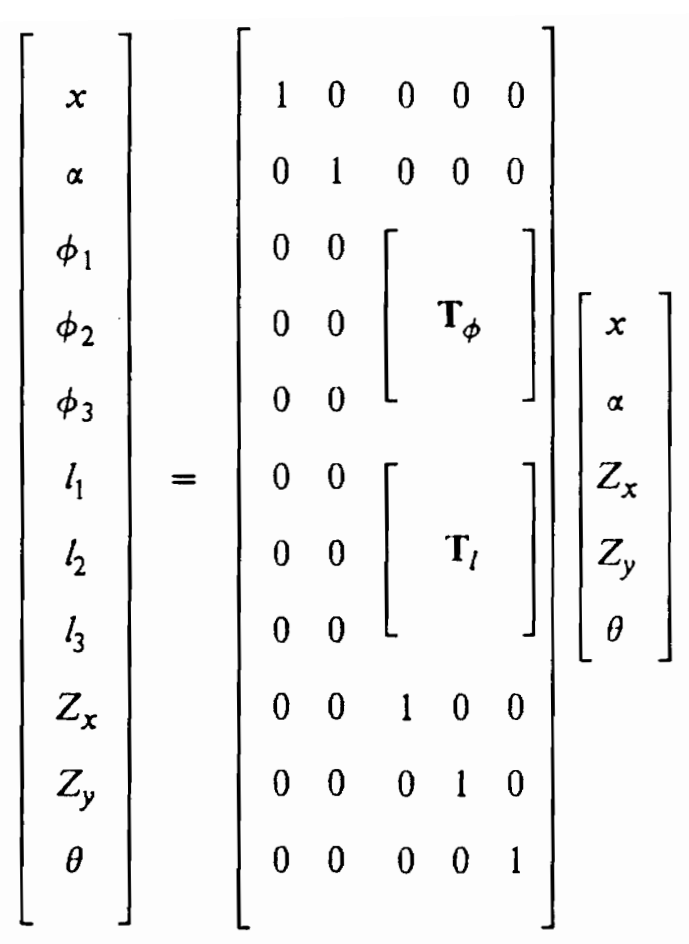

Where the transformation matrix, once again called $\mathbf{A}$, is exact for velocities and is a linear approximation for accelerations. Applying the transformation to the kinetic energy leads to

$$
T=\dot{\operatorname{Pr}}_{T}(t)^{t} \mathbf{A}^{t} \mathbf{M}_{\text {dep }} \mathbf{A} \dot{\operatorname{Pr}}_{T}(t)
$$

And the mass matrix for the actuator can be written in terms of independent coordinates as

$$
\mathbf{M}_{\text {acruator }}=\mathbf{A}^{t} \mathbf{M}_{\text {dep }} \mathbf{A}
$$

where $\mathbf{M}_{\text {acruator }}$ is a $5 \times 5$ matrix. Applying Lagrange's equation, Eqs. 3.31 and 3.33 , to Eq. 4.30 , the equations of motion become

$$
\mathbf{M}_{\text {acruator }} \ddot{\mathbf{P r}}_{T}=-\mathbf{R}_{F M}
$$

Adding the motor model used previously, the equations of motion for the planar truss proof mass actuator become

$$
\mathbf{M}_{\text {acruator }} \ddot{\mathbf{q}}_{T}+\mathbf{C}_{\text {actuator }} \dot{\mathbf{q}}_{T}=-\mathbf{R}_{F M}+\mathbf{F} \mathbf{V}_{m}
$$


The beam and actuator will now be combined. In this case the beam model is the same as that used for the proof mass and reaction wheel actuators. The actuator and beam can be combined by first augmenting the actuator equations by adding zeros, solving both sets of equations for the reactions, and setting them equal to one another. The overall system equations become

$$
\left(\mathbf{M}_{\text {acruator }}+\mathbf{M}_{\text {beam }}\right) \ddot{\mathbf{q}}+\mathbf{C}_{\text {acruator }} \dot{\mathbf{q}}+\mathbf{K}_{\text {beam }} \mathbf{q}=\mathbf{F V}_{m}
$$

where, for this model,

$$
\mathbf{q}=\left\{y_{1} \theta_{1} \ldots y_{4} \theta_{4} Z_{x} Z_{y} \theta\right\}^{t}
$$




\subsection{Basis for Comparison}

It has been mentioned several times so far that the purpose of this work is to compare the effectiveness of four actuators in their ability to damp the planar vibrations of a beam. At first sight that seems like a straight-forward task, but upon closer inspection several difficulties begin to appear. When comparing two subjects one must have some basis to start from in order to insure that (1) the comparison is fair, and (2) the conclusions reached are drawn from relevant data. In this comparison then, before any simulations were performed, a basis was formulated. In the descriptions of the actuator models, the basis for comparison was alluded to when discussing the inertias of the proof mass and reaction wheel, and the gains of the proof mass' lead screw and the reaction wheel's gear. In this section the basis for comparison will be described in detail to show that an attempt was made to compare the actuators without giving one an unfair advantage, and to show that the qualities compared are relevant to the overall goal of the work.

\subsection{Phvsical Parameters}

The idea behind making the comparison fair is to set up all the actuators as nearly identical as possible while still maintaining their individual uniquenesses. It is obvious, then, that each actuator's configuration cannot be altered, because that is where the uniqueness lies. A reaction wheel actuator is still a reaction wheel actuator for any motor-gear-reaction wheel set, but once the configuration is changed (for example, if it no longer applies moments, but rather forces) it can no longer be grouped with the class of actuators called reaction wheels. An important point to be gained from this, then, is that this study focuses not on any specific actuator set-up, (such as the 
LMED or AMCD referred to in the Literature Review ) but rather on how each actuator configuration and damping method fares in suppressing the vibrations of a beam. With those thoughts in mind, we can look at those physical variables which can be adjusted to insure a fair comparison.

Each actuator uses a motor (or motors) to produce the actuating forces. All motors are assumed to be the same in every actuator. Looking at the actuator illustrations in Fig. I quickly shows that the planar truss and the planar truss proof mass actuators have three "active parts" while the proof mass and the reaction wheel actuators have only one active part. To make up for this disadvantage three proof mass and three reaction wheel actuators are placed on the beam at different locations to give each actuating system the same number of active parts.

The proof mass actuator could acquire a distinct advantage by having a very large secondary mass (and likewise for the reaction wheel actuator). To account for this, the magnitudes of the secondary mass and the reaction wheel inertia are made equal. The magnitude of the secondary mass in the planar truss proof mass actuator is more difficult to pin down because this case deals with a combination proof mass/reaction wheel located at a single point rather than three proof masses or reaction wheels spaced along the beam. Taking that into consideration, the solution was to make the secondary mass in the planar truss proof mass actuator slightly less than three times that of a single proof mass, and it's mass moment of inertia slightly less than three times that of a single reaction wheel. This choice makes up for the disadvantage of being placed at a single location, but doesn't drastically alter the mode shapes of the beam.

Another place where mechanical advantage can be found is in the gains of the ball screws and gears found in the actuators. These are set equal to one another; the ball screw gains in the proof mass, planar truss, and planar truss proof mass actuators are equal to the gear ratios in the reaction wheel actuators.

The physical constants used in this work are shown in Appendix A. 


\subsection{Control Law}

Besides physical parameters which can be varied in the models, the control law also has a great effect on an actuator's performance. In order to be consistent throughout this study, a full state optimal control law is used for all actuators. For a linear system, the optimal control is a time-varying function of the system states. It can be shown that under certain conditions, the optimal control becomes time-invariant, and a constant set of feedback gains, the Kalman gains, can be derived. This class of control problems is known as the lincar quadratic regulator (LQR) problem. Its formulation is shown below. (The following discussion can be found in more detail in Kirk (25).)

The linear quadratic regulator problem can be proposed as:

Minimize the performance measure

$$
J=\frac{1}{2} \mathbf{x}^{T}\left(t_{f}\right) \mathbf{H} \mathbf{x}\left(t_{f}\right)+\frac{1}{2} \int_{t_{0}}^{t_{f}}\left[\mathbf{x}^{T}(t) \mathbf{Q}(t) \mathbf{x}(t)+\mathbf{u}^{t}(t) \mathbf{R}(t) \mathbf{u}(t)\right] d t
$$

subject to the following constraints:

$$
\dot{\mathbf{x}}(t)=\mathbf{A}(t) \mathbf{x}(t)+\mathbf{B}(t) \mathbf{u}(t)
$$

where the constraints are, of course, the system equations of motion in state space form. In the performance measure, $\mathbf{H}, \mathbf{Q}$, and $\mathbf{R}$ are "penalty matrices" which place indirect limits on the final states, the states during control, and the control values, respectively. This performance measure describes the desire to maintain the state vector as close to the origin as possible without expending excessive control effort. By applying a variational approach to this problem, the optimal control can be found to be a linear, time-varying function of the system states, Eq. 4.3.

$$
\mathbf{u}(t)=-\mathbf{R}^{-1}(t) \mathbf{B}^{T}(t) \mathbf{S}(t) \mathbf{x}(t)
$$

It can be shown that the matrix $\mathrm{S}$ satisfies the matrix differential equation (the Riccati equation) 


$$
\dot{\mathbf{S}}(t)=-\mathbf{S}(t) \mathbf{A}(t)-\mathbf{A}^{T}(t) \mathbf{S}(t)-\mathbf{Q}(t)+\mathbf{S}(t) \mathbf{B}(t) \mathbf{R}^{-1}(t) \mathbf{B}^{T}(t) \mathbf{S}(t)
$$

with the boundary condition $\mathbf{S}\left(t_{f}\right)=\mathbf{H}$. Kalman has shown that if

1) the plant is completely controllable

2) $\mathbf{H}=\mathbf{0}$

3) $\mathbf{A}, \mathbf{B}, \mathbf{Q}$, and $\mathbf{R}$ are constant matrices,

then $\mathbf{S}(t) \rightarrow \mathbf{S}$ (a constant matrix) as $t_{f} \rightarrow \infty$. This means that the optimal control law is no longer time varying, and can be written as

$$
\mathbf{u}(t)=-\mathbf{R}^{-1} \mathbf{B}^{T} \mathbf{S} \mathbf{x}(t)
$$

or

$$
\mathbf{u}(t)=-\mathbf{K} \mathbf{x}(t)
$$

For this work $\mathbf{H}$ is set to zero, and $\mathbf{Q}$ and $\mathbf{R}$ are constant, diagonal matrices. (The diagonal elements of $\mathbf{Q}$ are 10,000 , and those for $\mathbf{R}$ are 1 . This choice of penalties means that any offset of the states from zero is severely penalized while the control voltages can be fairly high.) The optimal control law was found by assuming $t_{f} \rightarrow \infty$, so Eqs. 5.5 and 5.6 apply, and the feedback gain matrix, $K$, is constant.

\subsection{Performance Indices}

Using the above control law provides a simple means for evaluating the effectiveness of each actuator, namely, the performance index $\mathrm{J}$. During simulations $\mathrm{J}$ can be calculated for each actuator system and then can be used to compare to other actuators. Perhaps a better, more meaningful index of performance is the power consumed by the actuators during control. For 
practical purposes, the power consumed by each actuator may be the limiting factor in actual control situations. The performance index, $\mathrm{J}$, does not adequately represent power. Where $\mathrm{J}$ is a function of the motor input voltage, it should be a function of both the armature voltage and current. A proper representation of the power consumed by any actuator system (since all actuators have the same motor models) in this study is

$$
J_{P}=\int_{t=0}^{t=t_{f}} \sum_{k=1}^{3} v_{k}(t) i_{k}(t) d t
$$

where $k=1,2,3$ denote the three motors in each system, and $v_{k}$ and $i_{k}$ are the armature voltage and current in each motor. This perhaps more practical performance index is also calculated during each simulation for each actuator system.

A third performance index which may offer some insight when sifting through the data to find the best actuator is the damping factor added to the beam by the actuators. This is ordinarily calculated from the logarithmic decrement of the beam's controlled response, but the responses obtained for these simulations are not conducive to accurate damping factor determination by the logarithmic decrement method. The reason is that the control law seems to exchange energy between modes in the system, resulting in "unclean" plots, and also the control is saturated part of the time, so the output is not linear over the entire response. Another performance index was used which represents the damping added to the beam. Integrating the energy in the system over time provides a relative "damping factor" which can be used to compare one actuator to another. This performance index can be written as:

$$
J_{D}=\int_{t=0}^{t=t_{f}} E d t
$$

where $E$ is the total system energy. 
All three of these performance indices are tabulated with the results of the simulations, and are used in the discussion of the results. 


\subsection{Simulation Results}

\subsection{ACSL Program}

The program used to simulate the response of the beam/actuator systems is called Advanced Continuous Simulation Language (ACSL), by Mitchell and Gauthier. This is a numerical integration program which provides a variety of integration methods to fit the size or speed of the system being modelled. The integration method used to do the bulk of the work in this project was the 2 nd order Runge Kutta constant time step routine. What follows is a discussion of the results obtained from the simulations of the various beam/actuator models using the abovementioned integration routine.

\subsection{Full Comparison of Four Actuators}

The beam/actuator systems were set up as described in the Basis for Comparison section, and the response of each system to initial conditions was simulated. The initial conditions imparted on the beam in the first set of simulations are shown in Fig. 10. This set of initial conditions is a linear combination of modes 1-8 (all modelled modes). The amplitudes were chosen such that each mode contributes $20 \mathrm{~J}$ to the potential (strain) energy in the beam. Overall, then, the beam sees a total of $160 \mathrm{~kJ}$ of potential energy (all strain) at time $t=0$. To give some feel for what this means, a first mode deflection with $20 \mathrm{~J}$ of strain energy is equivalent to a tip deflection of 0.089 meters. 


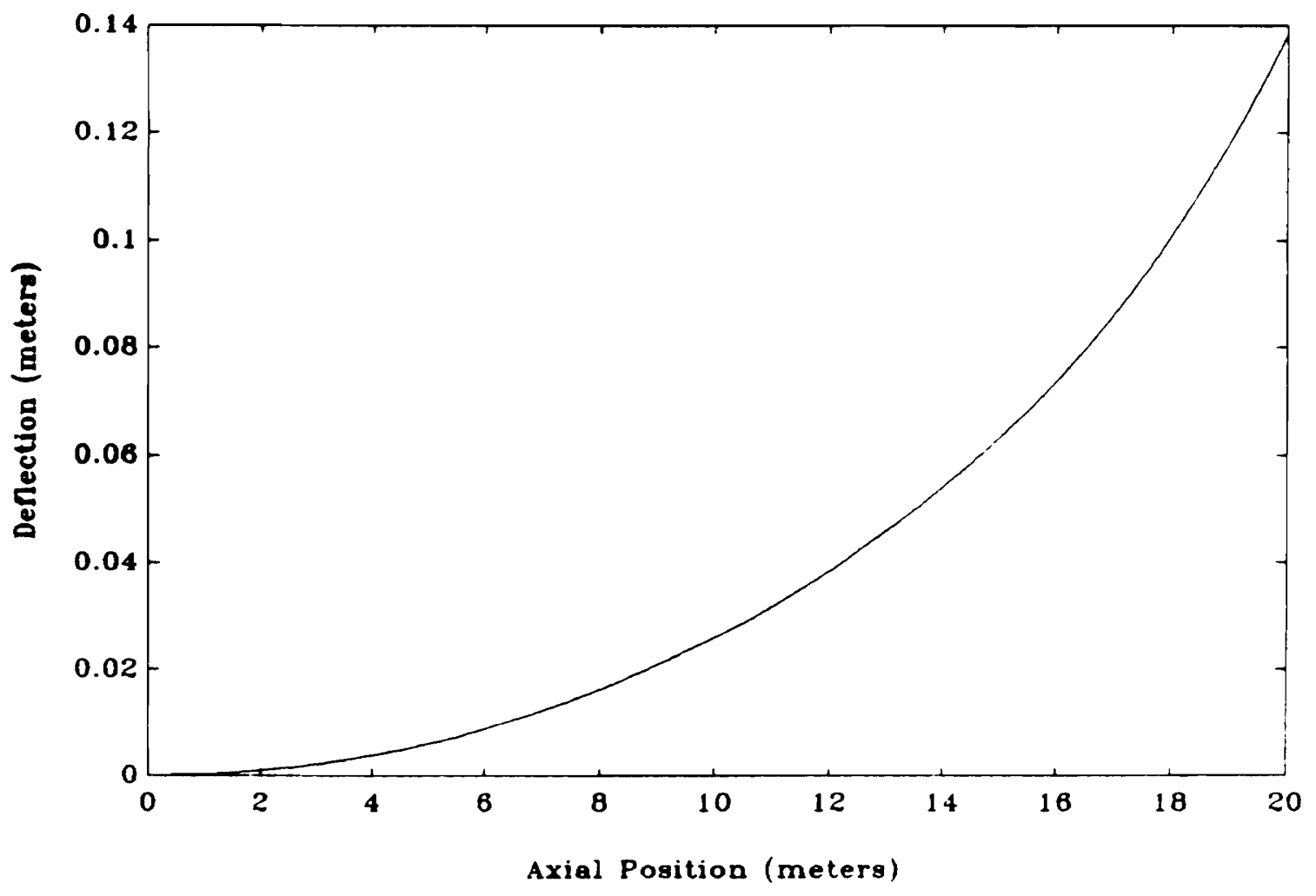

Figure 10. General Shape of Beam Initial Conditions Used in Simulations 
The same energy in higher modes corresponds, of course, to smaller nodal deflections, explaining why the initial conditions in Fig. 10 look largely like first mode. The reason for implementing the above-mentioned initial conditions was to excite all modes of vibration so as not to give one actuator an advantage over the others if it is particularly adept at damping a given mode.

The results of the simulations for each actuator are shown in Figs. 11-14. Each figure shows two plots; the first shows the energy of the beam versus time, and for a more "seeable" illustration, the second plot shows the tip deflection of the bcam versus time. Note that the time scale on the planar truss' plot is different from the others, it covers 5 seconds where the others cover 20 seconds. Figure 15 shows the motion of the planar truss actuator to provide a better idea of how this actuator works.

A second set of simulations was run to determine each actuator's effectiveness at damping 1st, 2nd, and 3rd mode initial conditions. In this case, each beam/actuator system was given initial conditions corresponding to $1 \mathrm{st}, 2 \mathrm{nd}$, or $3 \mathrm{rd}$ mode, and the response was simulated. The results of these simulations (and the previous simulations as well) are shown in Table 1. The performance indices in Table 1 are, as explained in the Basis for Comparison section, the LQR performance index, and the power consumed by the actuators during control. The last performance index is the time integral of the system energy, and will be discussed further in the next section. 


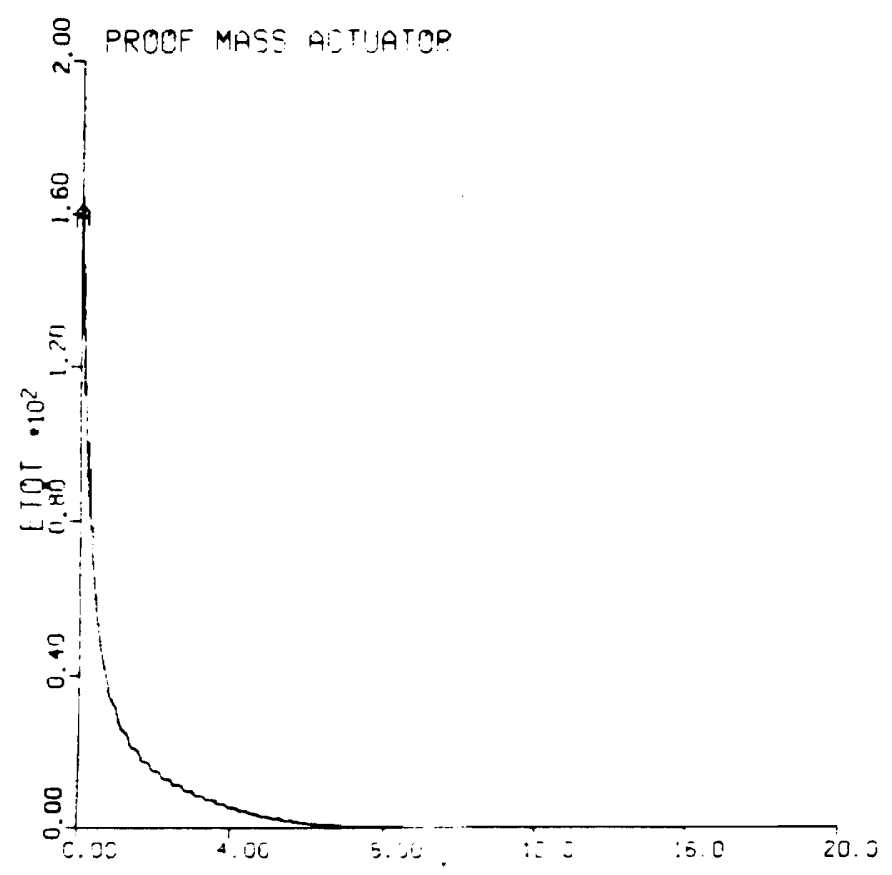

a. System Energy (Joules) vs. Time (sec)

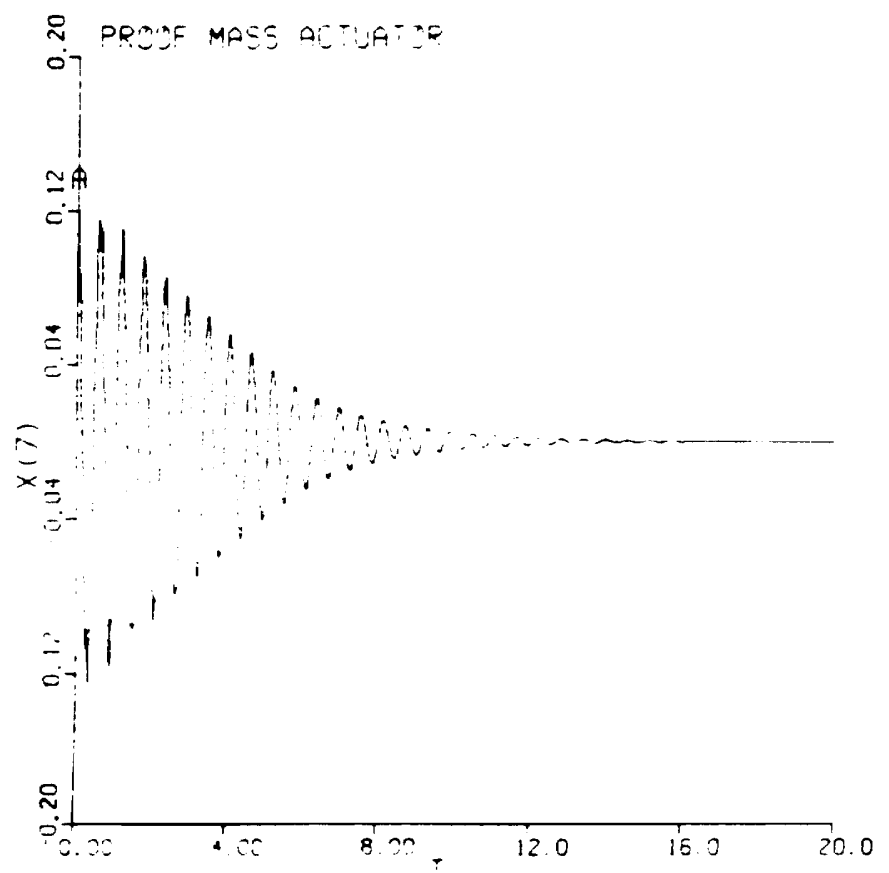

b. Beam Tip Deflection (meters) vs. Time (sec)

Figure 11. Response of Mini-Mast/Proof Mass Actuator System to Initial Conditions 


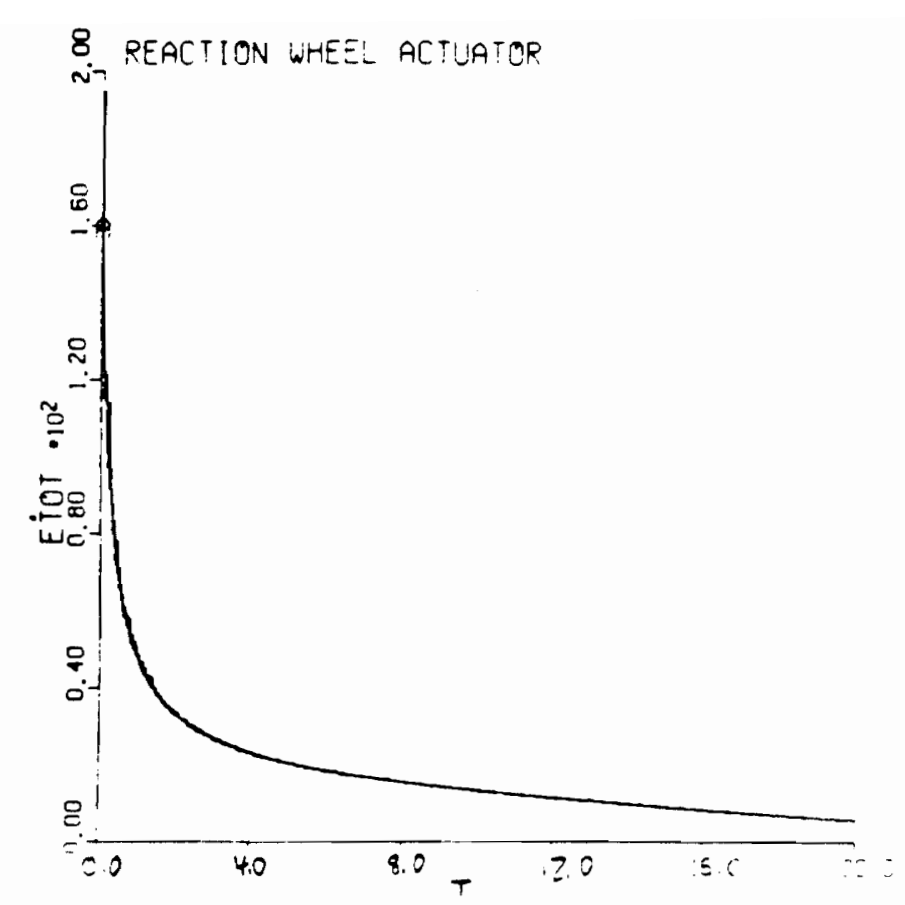

2. System Energy (Joules) vs. Time (sec)

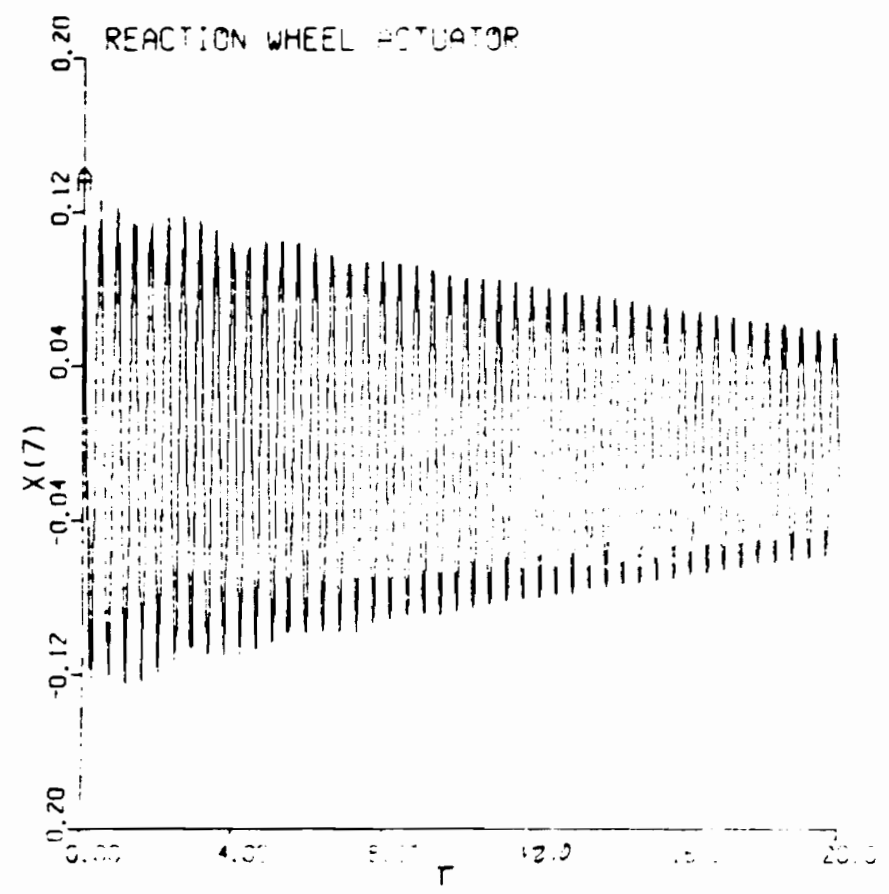

b. Beam Tip Deflection (meters) vs. Time (sec)

Figure 12. Responsc of Mini-Mast/Reaction Wheel Actuator System to Initial Conditions 


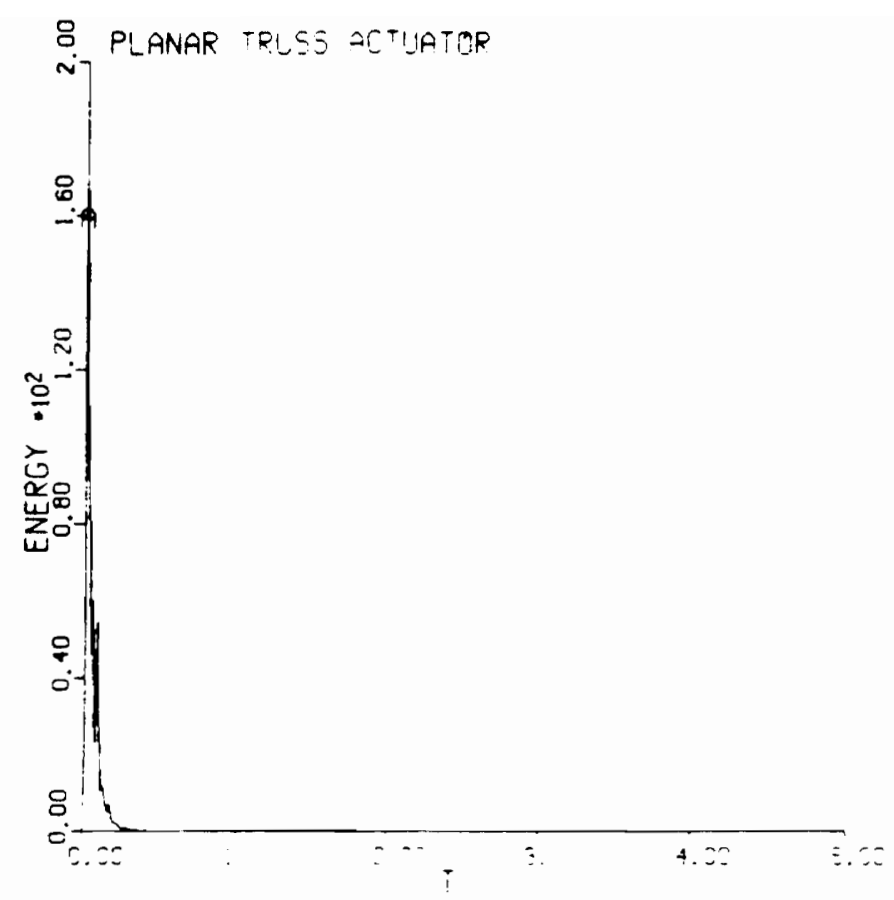

2. System Energy (Joules) vs. Time (sec)

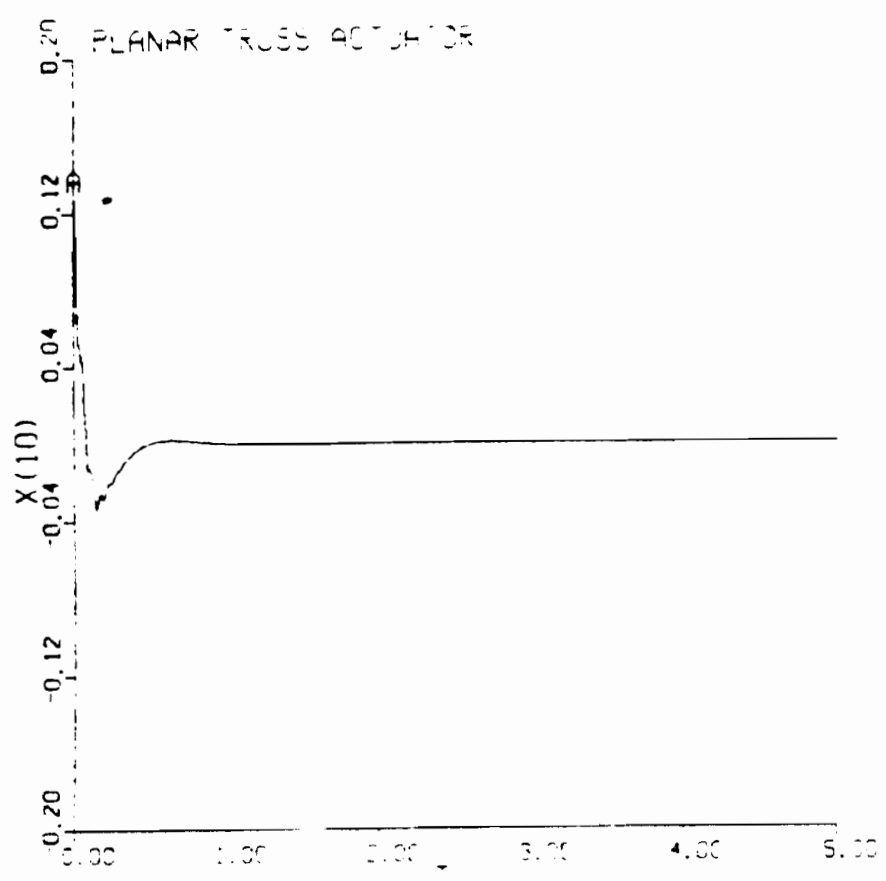

b. Beam Tip Deflection (meters) vs. Time (sec)

Figure 13. Response of Mini-Mast/Planar Truss Actuator System to Initial Conditions 


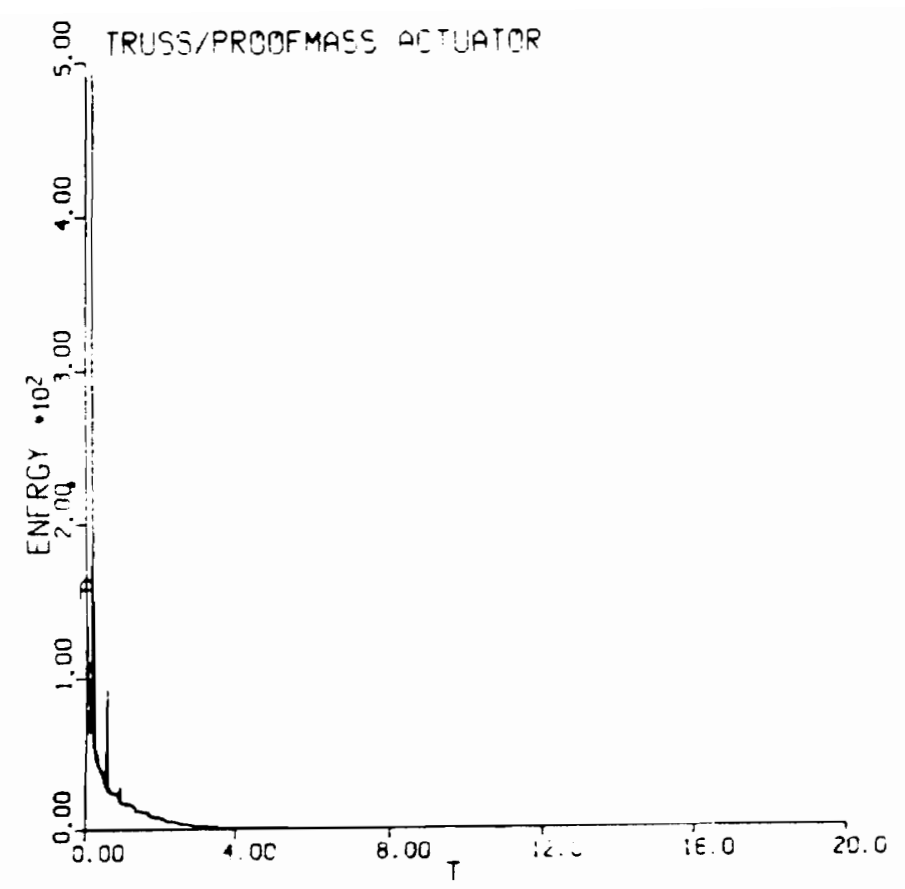

2. System Energy (Joules) rs. Time (sec)

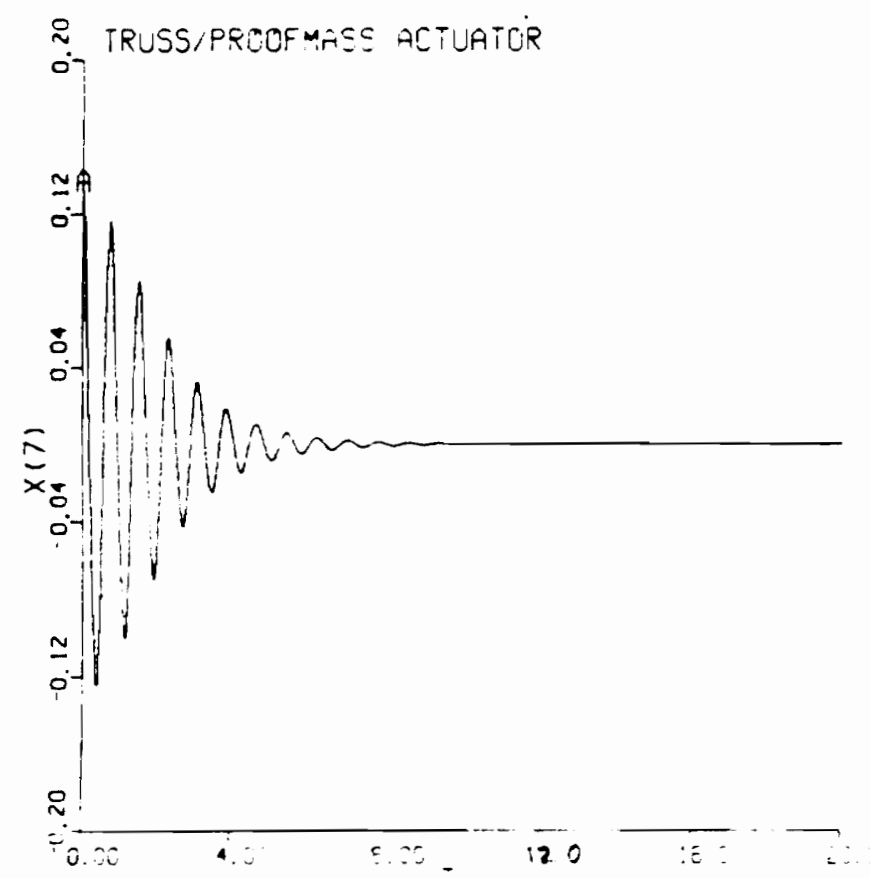

b. Beam Tip Deflection (meters) vs. Time (sec)

Figure 14. Response of Mini-Mast/Planar Truss Proof Mass Actuator System to Initial Conditions 


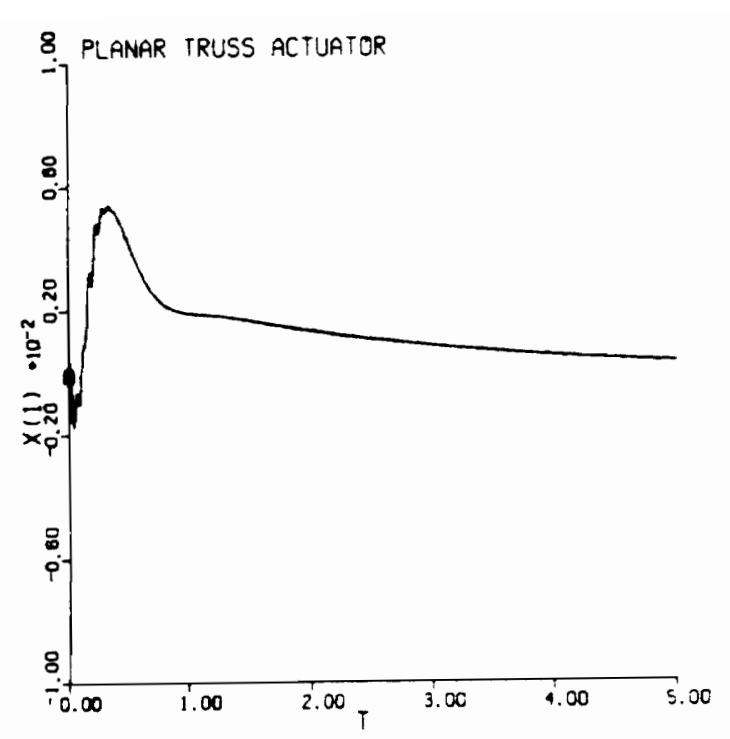

a. Motion of Planar Truss-- $Z_{x}$ vs. Time

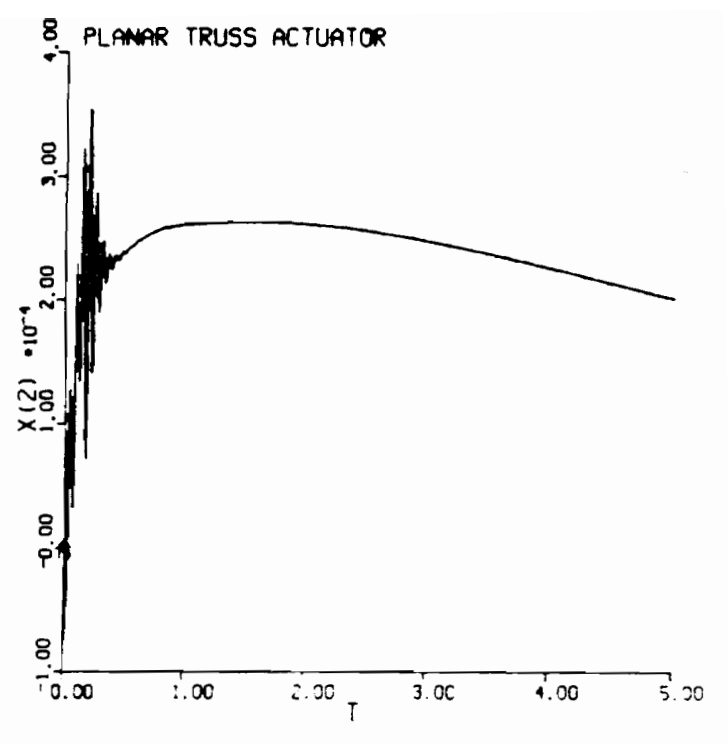

b. Motion of Planar Truss-- $Z$, vs. Time

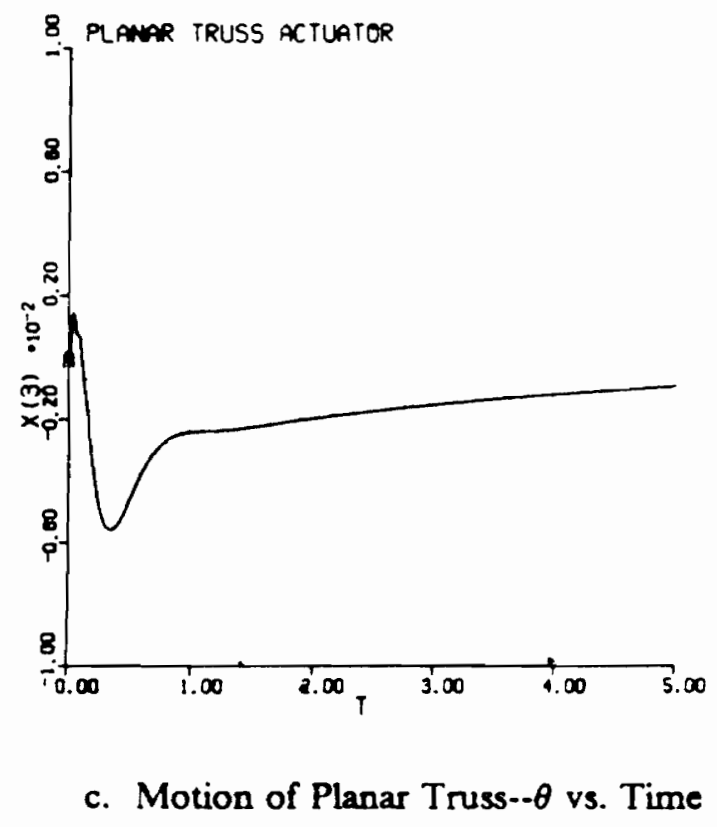

Figure 15. Motions of Planar Truss Actuator During Control 
Table 1. Results of Multiple Actuator Comparison

\begin{tabular}{|c|c|c|c|c|}
\hline Actuator & $\begin{array}{c}\text { *Initial } \\
\text { Conditions }\end{array}$ & $\begin{array}{c}\text { LQR Performance } \\
\text { Index } \\
\boldsymbol{J}\end{array}$ & $\begin{array}{c}\text { Energy Consumed } \\
\text { By Actuators } \\
\boldsymbol{J}_{\boldsymbol{P}} \text { (Joules) }\end{array}$ & $\begin{array}{c}\text { System Energy } \\
\text { Performance Index } \\
\boldsymbol{J}_{D} \text { (Joule-sec) }\end{array}$ \\
\hline \hline \multirow{3}{*}{ Proof } & 1st Mode & 103,000 & 76.3 & 347. \\
Mass & 2nd Mode & 21,000 & 94.2 & 66.8 \\
& 3rd Mode & 9,020 & 86.7 & 23.1 \\
& All Modes & 39,800 & 130. & 113. \\
\hline \multirow{3}{*}{ Reaction } & 1st Mode & 530,000 & 95.0 & 2630 \\
Wheel & 2nd Mode & 93,900 & 96.7 & 195. \\
& 3rd Mode & 18,900 & 89.2 & 39.6 \\
All Modes & 41,400 & 128. & 406. \\
\hline \multirow{3}{*}{ Proof Mass } & 1st Mode & 32,800 & 92.6 & 183. \\
& 2nd Mode & 18,000 & 778. & 29.4 \\
& 3rd Mode & 6,350 & 236. & 72.7 \\
\hline \multirow{3}{*}{ All Modes } & 19,400 & 93.5 & 8.75 \\
& 1st Mode & 5,640 & 137. & 30.2 \\
& 2nd Mode & 6,010 & 501. & 8.4 \\
\hline & 3rd Mode & 5,150 & 427. & \\
\hline
\end{tabular}

* Single mode initial conditions imparted $100 \mathrm{~J}$ of strain energy to the beam; multiple mode initial conditions imparted $160 \mathrm{~J}$ of strain energy to the beam ( $20 \mathrm{~J}$ for each modelled mode). 


\subsection{Discussion}

This section will discuss the results shown in chapter 6 . Fir $>t$, the results will be discussed to show their value in comparing actuator effectiveness. Next will follow a section discussing each performance index in relation to the others. Operational considerations of the actuators will be discussed to highlight actuator characteristics which are not pointed out in the simulation results. Finally, a section will be included to discuss extensions of this work, highlighting recommendations of where this work may lead.

\subsection{Discussion of Multiple Actuator Comparison Results}

The response plots shown in Figs. 11-14 show that the Variable Geometry Truss actuators do a better job of damping the vibrations of a beam excited by initial conditions. The plots of beam tip deflection provide a qualitative indication of how the actuators perform. The system energy plots provide an even better indication of performance, but only after examining the entries in Table 1 can the true performance be realized. The third colunn shows the integral of the total system energy over time. So these numbers represent the arca under the energy curves shown in Figs. 11-14. (In Fig. $12 \mathrm{~b}$ the energy does not go to zero in 20 seconds, but the entry in Table 1 represents an integral which is not truncated before the system energy reaches zero.) From these numbers it is seen that all actuators do a better job of damping higher modes than lower modes. The planar truss actuator, however, is much better than the inertia-type actuators at damping first mode. This can be explained by the mode of operation. The inertia-ty 5 : actuators can only apply "damping forces" to the beam when the secondary mass is being accclerated. This acceleration can only last as long as the motor back emf permits; in a short periud of time the motor reaches a steady-state 
velocity, the secondary mass is no longer accelerated, and no force is applied to the beam. If that period of time is less than half the period of oscillation of the first mode of the beam, then the actuator can be effective for only part of the oscillatory cycle of first mode. In the planar truss actuator, though, it is not reaction forces that are important in damping the beam, but rather kinematic inputs to the beam. The planar truss actuator (and VGT's in general) can "catch" the beam to remove the potential (strain) energy, then move the beam back to zero in a rigid body fashion. This action is shown in the response plots for $Z_{x}, Z_{y}$, and $\theta$ of the planar truss actuator (Fig. 15). They move to an extreme position (with some oscillations along the way, due to higher modes), and then retum slowly to their zero position. So, in this case, the motors can still be effective even when they are operating at steady-state velocity, if the velocity is in the right direction. It should be noted here that the planar truss actuator/beam configuration used in this work (Fig. 1), with the actuator located between the beam and ground, may be more advantageous than placing the actuator at some position along the beam. This, however, has not been studied and is recommended for future work.

A final point to be drawn from the "System Energy" data in Table 1 is that the planar truss/proof mass actuator is particularly good at controlling first mode. Even though this is an inertia-type actuator, its configuration allows it to control first mode better than either the proof mass or the reaction wheel actuators alone.

Another important consideration in choosing an actuator is the amount of energy consumed by the actuator in doing the control task. This is particularly important in space applications where the available energy is limited. Table 1 shows that the inertia-type actuators are all very close in the amount of energy consumed in controlling any mode. The planar truss actuator, however, consumes significantly more energy than the inertia-type actuators. This can be explained by the fact that the planar truss actuator must affect rigid body motion on the beam, a process which consumes more energy than moving a secondary mass. Placing the planar truss actuator at some position along the truss should cause the energy required for control to decrease because the part of the beam being moved rigidly is decreased. This improvement will be offset, however, by an 
expected decrease in damping performance, as mentioned above. Once again, that situation has not been studied in this work.

\subsection{Performance Indices}

A Linear Quadratic Regulator control law was used in controlling each of these systems. This control law worked nicely for the purpose of a comparison, because even though the dynamics were different for each system (different $A$ matrix) there were constant penalties $(Q$ and $R$ ) for determining the feedback gains. An important question to ask is "Does this control law minimize a performance index which truly represents the physical problem?" The physical problem is, from the point of view of this work, to minimize the integral of the total energy in the system and the integral of the control over time. This can be described by the equation

$$
J_{E}=\int_{t=0}^{t=t_{f}}(T(t)+V(t)+P(t)) d t
$$

or

$$
J_{E}=\int_{t=0}^{t=t_{f}}\left[\dot{\mathbf{x}}^{t} \mathbf{M} \dot{\mathbf{x}}+\mathbf{x}^{t} \mathbf{K} \mathbf{x}+\mathbf{V}_{m}^{t} \mathbf{I}_{m}\right] d t
$$

where $V_{m}^{t} \mathbf{I}_{m}$ is the power supplied to the motors. The performance index for the Linear Quadratic Regulator is described in Eq. 5.1, rewritten here as:

$$
J=\frac{1}{2} \int_{t=0}^{t=t_{f}}\left[\mathbf{x}^{t} \mathbf{Q} \mathbf{x}+\mathbf{u}^{t} \mathbf{R} \mathbf{u}\right] d t
$$

These two performance indices are nearly proportional. They both contain a term which is proportional to the state squared, and some form of the motor voltage input squared. This can also 
be seen from Table 1 . The LQR performance index is roughly a linear combination of system energy and the energy consumed by the actuators. By placing the system mass and stiffness matrices in $\mathrm{Q}$, the motor characteristics in $\mathrm{R}$, and adding terms to $\mathrm{Q}$ (which are multiplied by the velocities of the motors) to account for the motor armature current, Eq. 7.2 could very nearly be represented by Eq. 7.3. A point to be gained from this is that for a vibration control problem with the objectives of high damping and low power requirements, the LQR control law with diagonal $\mathrm{Q}$ and $\mathrm{R}$ works nicely, but perhaps a better optimal control law would be one that minimizes the performance index in Eq. 7.3.

\subsection{Actuator Operational Considerations}

There are several points concerning various actuator characteristics which should be considered when comparing one actuator to another. These points are not drawn directly from the simulation results, but have an impact on actuator design or on the choice of an actuator for a particular application.

The derivation in Chapter 4 showed that the reaction wheel actuator is completely analogous to the linear proof mass actuator with the exception that one provides moments to add damping to the beam while the other provides forces. This analogy holds true as long as the discussion remains in two dimensions. The reader should keep in mind, though, that if the beam is allowed to move out of the plane (consider, for example, torsion of the beam about its longitudinal axis) then the angular velocities of the reaction wheel bring about gyroscopic effects which make the model more complicated. The two dimensional results presented in this paper do not, then, provide an entirely clear picture of the total dynamics of the reaction wheel. Another point along those same lines is that the secondary mass of the proof mass actuator has a varying mass moment of inertia (calculated about an axis fixed to the beam). That moment of inertia is held constant in the proof mass actuator model in this work (a valid assumption, considering the small motions of 
the secondary mass), but for some actuators which see larger secondary mass motions (such as the LDCM and LMED) it's effects on the system may need to be considered.

An important characteristic of the planar truss actuator is that it is an extension of the Mini-Mast by one bay, with the difference that the links making up the actuator are active. This active bay could be located anywhere along the beam, which makes the planar truss actuator very flexible for applications such as pointing or shape control. Global beam motions can be applied and controlled by the actuator to accomplish tasks such as positioning solar arrays or antennas. Global motions cannot be applied by the inertia-type actuators. Another advantage of the planar truss is that it does not require the added mass necessary for the operation of inertia-type actuators. That added mass will be costly when these ideas are implernented in space.

The conclusion to be drawn from this discussion is that, from an operational standpoint, the planar truss actuator is more favorable than the inertia-type actuators. It allows for global positioning (slewing, shape control) of the beam, it does not come with the penalty of an added secondary mass, and as was shown in the previous section, the planar truss actuator is very effective in controlling vibrations of a flexible beam.

\subsection{Recommendations}

Throughout this discussion several areas of this work have been mentioned which deserve further consideration. First, this is a planar study only. An obvious extension of this work is to study the effectiveness of actuators controlling a beam in three dimensions. This could point out effects which are unseen in a planar study, such as gyroscopic effects of the reaction wheel actuators. A second area of interest is a study of systems which are free-floating in space. These are more realistic space situations which are no longer attached to ground through either a beam or an actuator. Another important extension of this work is to study the effects of actuator placement on the beam. It was mentioned earlier that placing the planar truss actuator at a position along the beam should be studied, but optimizing the position of the other actuators should be looked at as 
well. A final recommendation is to study the use of a modified control law, such as one using the performance index shown in Eq. 7.2. 


\section{$\underline{\text { References }}$}

1. Strunce, R.R., and Carman, R.W., "Active Control of Spite Structures (ACOSS): A Status Report," Structures, Structural Dynamics, and Materials ( onference, 25th, Palm Springs, CA, May 14-16, 1984, Technical Papers, Part 2, pp.348-356. AIAA Paper 84-1027.

2. Balas, M.J., "Trends in Large Space Structure Control Theory: Fondest Hopes, Wildest Dreams," IEEE Transactions of Automatic Control, Vol. AC-27, No. 3, June 1982, pp.522-535.

3. Kosut, R.L., Salzwedel, H., and Emami-Naeini, A., "Robust Control of Flexible Spacecraft," Journal of Guidance and Control, Vol. 6, No. 2, .March-April 1983, pp. 104-111.

4. Margulies, G., and Aubrun, J.N., "Geometric Theory of Single-Gimbal Control Moment Gyro Systems," The Journal of the Astronautical Sciences, Vol. XXVI, No. 2, April-June 1978, pp. 159-191.

5. Aubrun, J.N., "Analytical and Experimental Research in Large Space Structures Control," AIAA 23rd Aerospace Sciences Meeting, Reno, Nevada, January 14-17, 1985, pp. 1-15, AIAA Paper 85-0356.

6. Anderson, W.W., and Groom, N.J., "The Annular Momentum Control Device (A.MCD) and Potential Applications," NASA TN D-7866, .March 1975.

7. Joshi, S.M., and Groom, N.J., "Modal Damping Enhancement in Large Space Structures Lsing A.MCD's," Journal of Guidance and Control, Vol. 3, No. 5, September-October 1980, pp. 477-479.

8. Zimmerman, D.C., Inman, D.J., and Homer, G.C., "Dynamic Characterization and Microprocessor Control of the NASA/LVA Proof Mass Actuator," Structures, Structural Dynamics, and Materials Conference, 25th, Palm Springs, CA, May 14-16, 1984, Technical Papers, Part 2, pp. 573-577, AIAA Paper 84-1077.

9. Doane, G.B., Waites, H., and Edgemon, G.D., "Development and Use of a Linear Momentum Exchange Device," Procecdings of the First NASA/DOD Control/structures Technology Conference, Norfolk, VA, Nov. 18-21, 1986, pp. 431-440.

10. Ham, F.M., Hennings, B.L., and Greeley, S.W., Harris Corporation, AIAA Paper 87-2321. 
11. Mills, R.A., "Active Vibration Control of a Cantilevered Beam: A Study of Control Actuators," Proceedings of the 34th International Astronautical Congress, Budapest, Hungary, October $10-15,1983$.

12. Natori, M., Iwasaki, K., and Kuwao, F., "Adaptive Planar Truss Structures and Their Vibration Characteristics," Structures, Structural Dynamics, and Materials Conference, 28th, Monterey, CA, April 6-8, 1987, Technical Papers, Part 2B, pp. 125-134, AIAA Paper 87-0743.

13. Lovejoy, V.D., Robertshaw, H.H., Patten, W.N., and Horner, G.C., "Dynamics and Control of a Planar Truss Actuator," Vibration Control and Active Vibration Suppression, DE-Vol. 4, 1987, pp. 47-55.

14. Bailey, T., and Hubbard J.E. Jr., "Distributed Piezoelectric-Polymer Active Vibration Control of a Cantilever Beam," Journal of Guidance and Control, Vol. 8, No. 5, September-October, 1985, pp. 605-611.

15. Hanagud, S., Obal, M.W., and Calise, A.J., "Optimal Vibration Control By the Use of Piezoceramic Sensors and Actuators," Structures, Structural Dynamics, and Miaterials Conference, 28th, Monterey, CA, April 9-10, 1987, Technical Papers, Part 2B, pp.987-997, AIAA Paper 87-0959.

16. Fanson, J.L., and Chen, J-C., "Structural Control by the Use of Piezoelectric Active Members," Proceedings of the First NASA/DOD Control/Structures Interaction Technology Conference, Norfolk, VA, November 18-21, 1986, pp.809-829.

17. Edberg, D.L., "Control of Flexible Structures by Applied Thermal Gradients," AI $\mathrm{A}$ Journal, Vol. 25, No. 6, June 1987, pp. 877-883.

18. Johnson, C.R. Jr., "Adaptive Modal Control of Large Flexible Spacecraft," Journal of Guidance and Control, Vol. 3, No., 4, July-August 1980, pp.369-375.

19. Schafer, B., and Holzach, H., "Experimental Research on Flexible Beam Modal Control," Structures, Structural Dynamics, and Materials Conference, 25th, Palm Springs, CA, May 14-16, 1984, Technical Papers, Part 2, pp. 317-326, AIAA Paper 84-1020.

20. Wie, B., "Active Vibration Control Synthesis for the COFS-I: A Classical Approach," AIAA Guidance, Navigation, and Control Conference, Monterey, CA, August 17-19, 1987, Technical Papers, pp.361-369, AIAA Paper 87-2322.

21. Politansky, H., and Pilkey, W.D., "Suboptimal Feedback Vibration Control of a Beam With a Proof-Mass Actuator," AIAA Guidance, Navigation, and Control Conference, Monterey, CA, August 17-19, 1987, Technical Papers, pp. 370-378, AIAA Paper $87-2323$. 
22. Martinovic, Z.N., Haftka, R.T., Hallauer, W.L. Jr., and Schamel, G.C., "A Comparison of Active Vibration Control Techniques: Output Feedback Vs. Optimal Control," Structures, Structural Dynamics, and Materials Conference, 28th, Monterey, CA, April 6-8, 1987, Technical Papers, Part 2B, pp. 610-621, AlAA Paper 87-0904.

23. Meirovitch, L., Elements of Vibration Analysis, McGraw Hill, New York, N.Y., 1986.

24. Meirovitch, L., Methods of Analytical Dynamics, McGraw Hill, New York, N.Y., 1970.

25. Kirk, D.E., Optimal Control Theory, Prentice-Hall, Englewood Cliffs, N.J., 1970. 


\section{Appendix A. List of Physical Constants}

$\begin{array}{ll}m_{1} & 8 \mathrm{~kg} \\ m_{2} & 12 \mathrm{~kg} \\ I_{1} & 8 \mathrm{kgm}^{2} \\ I_{2} & 12 \mathrm{kgm}^{2} \\ J_{S M} & 20 \mathrm{~kg} \\ M_{S M} & 20 \mathrm{kgm} \\ K_{T} & 0.4237 \mathrm{~N}-\mathrm{m} / \mathrm{amp} \\ K_{B} & \text { Volt }-\mathrm{sec} \\ R_{a} & \text { Ohms } \\ G_{p} & 8.0851 E-4 \\ G_{r} & \end{array}$

Entries in Q 10,000 (all on diagonal)

Entries in $\mathbf{R} \quad 1$ (all on diagonal) 


\section{$\underline{\text { Vita }}$}

The author was born on April 10, 1963 in Clifton Forge, Virginia. He grew up in McClung, Virginia, spending many days on the farm, and many nights on the baseball field. He attended Bath County High School, graduating Valedictorian of his class in 1981. He entered Virginia Polytechnic Institute and State University in the Fall of 1981 majoring in Biology, and, after several years of searching, found a home Mechanical Lngineering, where he obtained a Bachelor of Science in 1986. After spending an enjoyable summer at home on the farm, he began graduate work at VPI\&SU, again in Mechanical Engineering, which has resulted in a Master of Science degree. The author will soon begin work on a PhD. The future beyond that is uncertain, however a modest herd of cattle (and maybe a few pigs) is not out of the question.

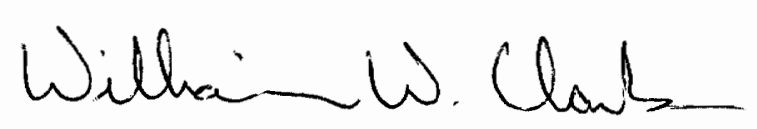

